\title{
UNBOUNDED DERIVED CATEGORIES OF SMALL AND BIG MODULES: IS THE NATURAL FUNCTOR FULLY FAITHFUL?
}

\author{
LEONID POSITSELSKI AND OLAF M. SCHNÜRER
}

\begin{abstract}
Consider the obvious functor from the unbounded derived category of all finitely generated modules over a left noetherian $\operatorname{ring} R$ to the unbounded derived category of all modules. We answer the natural question whether this functor defines an equivalence onto the full subcategory of complexes with finitely generated cohomology modules in two special cases. If $R$ is a quasi-Frobenius ring of infinite global dimension, then this functor is not full. If $R$ has finite left global dimension, this functor is an equivalence. We also prove variants of the latter assertion for left coherent rings, for noetherian schemes and for locally noetherian Grothendieck categories.
\end{abstract}

\section{INTRODUCTION}

Let $R$ be a left noetherian ring. Then the inclusion $\bmod (R) \subset \operatorname{Mod}(R)$ of the category of all finitely generated left $R$-modules into the category of all left $R$-modules is trivially fully faithful and exact. We ask when the induced functor

$$
\mathrm{D}(\bmod (R)) \rightarrow \mathrm{D}(\operatorname{Mod}(R))
$$

on unbounded derived categories is fully faithful as well and when its essential image is the subcategory $\mathrm{D}_{\bmod (R)}(\operatorname{Mod}(R)) \subset \mathrm{D}(\operatorname{Mod}(R))$ of complexes with cohomology objects in $\bmod (R)$. Put together, we study the question when the induced functor

$$
F: \mathrm{D}(\bmod (R)) \rightarrow \mathrm{D}_{\bmod (R)}(\operatorname{Mod}(R))
$$

is an equivalence. We present a negative and two positive results. Before explaining these results, we would like to mention that the answer to the corresponding question in the bounded above case is well-known: The induced functor on bounded above derived categories is always an equivalence $\mathrm{D}^{-}(\bmod (R)) \stackrel{\sim}{\rightarrow} \mathrm{D}_{\bmod (R)}^{-}(\operatorname{Mod}(R))$ (special case of Proposition 2.1).

The negative result is that $F$ is neither full nor essentially surjective (not even up to direct summands) if $R$ is a quasi-Frobenius ring (= a left noetherian, left self-injective ring) of infinite global dimension (Theorem 3.1). Quasi-Frobenius rings are always left and right noetherian and left and right artinian. The ring $k[\varepsilon] /\left(\varepsilon^{2}\right)$ of dual numbers over a field $k$ and $\mathbb{Z} / 4 \mathbb{Z}$ are examples of commutative quasi-Frobenius rings of infinite global dimension.

The first positive result is that $F$ is an equivalence whenever $R$ has finite left global dimension (Corollary 4.5). Commutative examples of such rings are polynomial rings $k\left[x_{1}, \ldots, x_{n}\right]$ over a field $k$ and all regular quotients of such rings. In fact, Corollary 4.5 is the slightly stronger statement that $F$ is an equivalence whenever $R$ is a left coherent ring of finite left global dimension and $\bmod (R)$ denotes the category of all finitely presented left $R$-modules.

2020 Mathematics Subject Classification. Primary 18G80; Secondary 16E35, 16L60, 18G20, 18E10.

Key words and phrases. Unbounded derived category, quasi-Frobenius ring, locally noetherian Grothendieck category, absolute derived category. 
Of course there are many examples of left noetherian rings which have infinite left global dimension and are not left self-injective, even if one restricts attention to finitedimensional algebras over a field or to commutative noetherian rings. It would be nice to characterize, in terms of classical homological algebra, all left noetherian (or coherent) rings $R$ for which $F$ is an equivalence.

The second positive result is a generalization of the first positive result to algebraic geometry. If $X$ is a regular noetherian scheme of finite Krull dimension, then the functor induced by the inclusion $\operatorname{coh}(X) \subset \mathrm{Q} \operatorname{coh}(X)$ is an equivalence

$$
\mathrm{D}(\operatorname{coh}(X)) \stackrel{\sim}{\rightarrow} \mathrm{D}_{\operatorname{coh}}(\mathrm{Q} \operatorname{coh}(X))
$$

(Corollary 5.12).

Similarly as above, it would be nice to characterize all noetherian schemes $X$ for which the obvious functor

$$
E: \mathrm{D}(\operatorname{coh}(X)) \rightarrow \mathrm{D}_{\text {coh }}(\mathrm{Q} \operatorname{coh}(X))
$$

is an equivalence. Again, the bounded above case is a classical result: The functor $\mathrm{D}^{-}(\operatorname{coh}(X)) \stackrel{\sim}{\rightarrow} \mathrm{D}_{\text {coh }}^{-}(\mathrm{Q} \operatorname{coh}(X))$ is always an equivalence ([SGA6, Exposé II, Proposition 2.2.2, p. 167], [SP20, 0FDA]). Our negative result shows that there are many affine schemes $X$, for example Spec $k[\varepsilon] /\left(\varepsilon^{2}\right)$ and $\operatorname{Spec} \mathbb{Z} / 4 \mathbb{Z}$, such that $E$ is not an equivalence.

Our two positive results are in fact consequences of abstract categorical results. Assume that $\mathcal{A}$ is an abelian category and that $\mathcal{U} \subset \mathcal{A}$ is an abelian subcategory closed under extensions. Then it is natural to ask when

$$
G: \mathrm{D}(\mathcal{U}) \rightarrow \mathrm{D}_{\mathcal{U}}(\mathcal{A})
$$

is an equivalence. If $\mathcal{A}$ has finite global dimension and some technical conditions are satisfied, the answer to this question is affirmative (Theorems 4.3 and 5.10; Corollary A.2 in the hereditary case).

An important special case where $G$ is an equivalence is the case that $\mathcal{A}$ is a locally noetherian Grothendieck category of finite global dimension and that $\mathcal{U} \subset \mathcal{A}$ is its full subcategory of noetherian objects (Corollary 5.11). Let us say a few words about the proof of this assertion. The argument consists of three steps.

Firstly, for any locally coherent Grothendieck category $\mathcal{A}$ and its full subcategory $\mathcal{U} \subset \mathcal{A}$ of coherent objects, the functor $G$ is essentially surjective provided that $\mathcal{A}$ has finite global dimension (special case of Proposition 4.2; note that $\mathcal{U}$ has finite global dimension by Proposition 2.1).

Secondly, for any abelian category $\mathcal{A}$, consider its so-called absolute derived category $\mathrm{D}^{\text {abs }}(\mathcal{A})$. Then, for any abelian category $\mathcal{A}$ of finite global dimension, the canonical functor $\mathrm{D}^{\text {abs }}(\mathcal{A}) \rightarrow \mathrm{D}(\mathcal{A})$ is an equivalence (Remark 5.4).

Thirdly, for any locally noetherian Grothendieck category $\mathcal{A}$, the obvious triangulated functor

$$
\mathrm{D}^{\mathrm{abs}}(\mathcal{U}) \rightarrow \mathrm{D}^{\mathrm{abs}}(\mathcal{A})
$$

is fully faithful (special case of Theorem 5.5). Notice that this holds for categories $\mathcal{A}$ of infinite global dimension as well. So, in this particular respect, absolute derived categories are better behaved than conventional unbounded derived categories.

If now $\mathcal{A}$ is a locally noetherian Grothendieck category of finite global dimension, then its subcategory $\mathcal{U}$ of noetherian objects has finite global dimension as well and we deduce 
from the above three steps and commutativity of the diagram

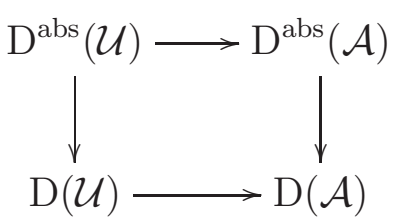

that $G$ is an equivalence.

Acknowledgment. We thank the organizers of the summer school Two weeks of silting in Stuttgart 2019 where we obtained our first negative result for the dual numbers. The second author thanks Ciprian Modoi for useful comments. We thank the referee for reading our text carefully and for spotting several small inaccuracies. We thank Pierre Schapira for providing references. The first author is supported by the GAČR project 20-13778S and research plan RVO: 67985840.

\section{Notation And Preliminaries}

Let $R$ be a left coherent ring. The abelian category of all left $R$-modules is denoted by $\operatorname{Mod}(R)$ and its full abelian subcategory of all finitely presented left $R$-modules by $\bmod (R)$. We just say module instead of left module in the following. The fully faithful, exact inclusion functor $\bmod (R) \rightarrow \operatorname{Mod}(R)$ between abelian categories induces a triangulated functor

$$
F: \mathrm{D}(\bmod (R)) \rightarrow \mathrm{D}(\operatorname{Mod}(R))
$$

between the corresponding unbounded derived categories. This functor certainly lands in $\mathrm{D}_{\bmod (R)}(\operatorname{Mod}(R))$, the full subcategory of $\mathrm{D}(\operatorname{Mod}(R))$ consisting of all objects $C$ with $\mathrm{H}^{i}(C) \in \bmod (R)$ for all $i \in \mathbb{Z}$. Since $F$ obviously commutes with cohomology, it reflects the zero object and hence, being a triangulated functor, is conservative (= reflects isomorphisms). We remind the reader that $\mathrm{D}(\operatorname{Mod}(R))$ has all coproducts and products and that these can be formed naively because coproducts and products of quasi-isomorphisms between complexes in $\operatorname{Mod}(R)$ are quasi-isomorphisms. Note also that $\mathrm{D}(\operatorname{Mod}(R))$ is idempotent-complete, and hence so is $\mathrm{D}_{\bmod (R)}(\operatorname{Mod}(R))$.

In the case of a left noetherian ring $R$, it is well-known that the functor induced by $F$ is an equivalence

$$
\mathrm{D}^{-}(\bmod (R)) \stackrel{\sim}{\rightarrow} \mathrm{D}_{\bmod (R)}^{-}(\operatorname{Mod}(R))
$$

of triangulated categories (e.g. [SP20, 0FCL], [Huy06, Proof of Proposition 3.5]); here D indicates the vanishing of all sufficiently high cohomologies. This assertion holds more generally for any left coherent ring $R$; this is a consequence of Proposition 2.1 below.

Let now $\mathcal{A}$ be an abelian category and let $\mathcal{U} \subset \mathcal{A}$ be a non-empty, full subcategory closed under kernels, cokernels, and extensions. Then $\mathcal{U}$ is an abelian category as well, and the fully faithful, exact inclusion functor $\mathcal{U} \rightarrow \mathcal{A}$ induces a triangulated functor

$$
\mathrm{D}(\mathcal{U}) \rightarrow \mathrm{D}(\mathcal{A})
$$

between the unbounded derived categories. Once again, this functor is conservative and lands in $\mathrm{D}_{\mathcal{U}}(\mathcal{A}) \subset \mathrm{D}(\mathcal{A})$, the full subcategory of all complexes in $\mathcal{A}$ with cohomology objects belonging to $\mathcal{U}$. One would like to know when $\mathrm{D}(\mathcal{U}) \rightarrow \mathrm{D}_{\mathcal{U}}(\mathcal{A})$ is an equivalence of categories. The following result is well-known (e.g. [KS94, Proposition 1.7.11] [KS06, Theorem 13.2.8]); we include its proof for convenience. 
Proposition 2.1. Let $\mathcal{A}$ be an abelian category and let $\mathcal{U} \subset \mathcal{A}$ be a non-empty, full subcategory closed under kernels, cokernels and extensions. Assume that for any epimorphism $A \rightarrow U$ in $\mathcal{A}$ with $U \in \mathcal{U}$ there exists a morphism $V \rightarrow A$ in $\mathcal{A}$ with $V \in \mathcal{U}$ such that the composition $V \rightarrow A \rightarrow U$ is an epimorphism. Then the obvious functor is an equivalence $\mathrm{D}^{-}(\mathcal{U}) \stackrel{\sim}{\rightarrow} \mathrm{D}_{\mathcal{U}}^{-}(\mathcal{A})$.

Remark 2.2. If we replace the assumption that the full subcategory $\mathcal{U}$ in Proposition 2.1 is closed under extensions by the assumption that it is closed under finite coproducts, it will automatically be closed under extensions. Indeed, let $S \hookrightarrow E \rightarrow Q$ be a short exact sequence in $\mathcal{A}$ with $S, Q \in \mathcal{U}$. By assumption, there is an object $F \in \mathcal{U}$ and a morphism $u: F \rightarrow E$ such that $F \stackrel{u}{\rightarrow} E \rightarrow Q$ is an epimorphism. We obtain a morphism

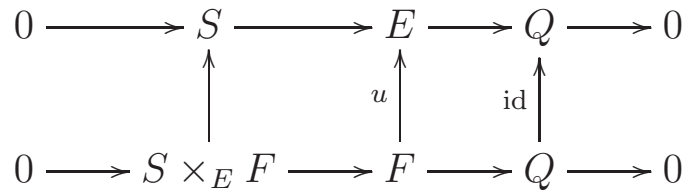

of short exact sequences. Note that $S \times_{E} F$ is in $\mathcal{U}$ as the kernel of $F \rightarrow Q$. The left square is cartesian but also cocartesian. Hence $E$ is the cokernel of the morphism $S \times_{E} F \rightarrow S \oplus F$ between objects of $\mathcal{U}$ and hence in $\mathcal{U}$.

Proof. Let $A$ be a complex in $\mathcal{A}$ with cohomologies $\mathrm{H}^{i}(A) \in \mathcal{U}$, for all $i \in \mathbb{Z}$. Let $N \in \mathbb{Z}$ and assume that $A^{i} \in \mathcal{U}$ for all integers $i>N$. The cocycle object $\mathrm{Z}^{N+1}(A)$ is in $\mathcal{U}$ as the kernel of $A^{N+1} \rightarrow A^{N+2}$. The coboundary object $\mathrm{B}^{N+1}(A)$ is then in $\mathcal{U}$ as the kernel of $\mathrm{Z}^{N+1}(A) \rightarrow \mathrm{H}^{N+1}(A)$. Therefore, by assumption, there is an object $U \in \mathcal{U}$ and a morphism $u: U \rightarrow A^{N}$ such that the composition $U \stackrel{u}{\rightarrow} A^{N} \rightarrow \mathrm{B}^{N+1}(A)$ is an epimorphism. Similarly, there is an object $V \in \mathcal{U}$ and a morphism $v: V \rightarrow \mathrm{Z}^{N}(A)$ such that the composition $V \stackrel{v}{\rightarrow} \mathrm{Z}^{N}(A) \rightarrow \mathrm{H}^{N}(A)$ is an epimorphism. The diagram

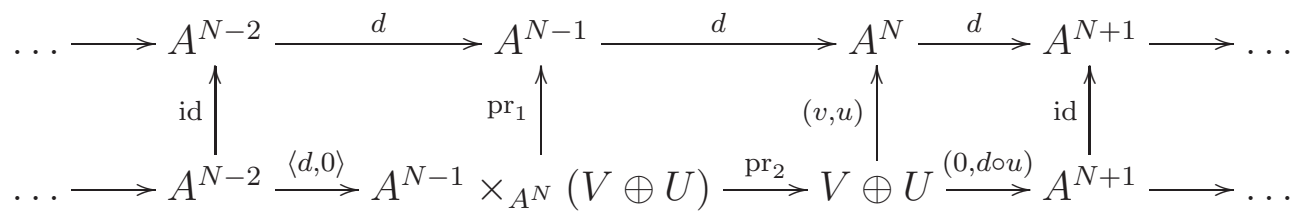

is clearly commutative and its lower row is a complex in $\mathcal{A}$. The obvious diagram chases together with the Freyd-Mitchell embedding theorem show that the vertical arrows define a quasi-isomorphism between the two rows. Note that the component $V \oplus U$ of the lower complex is in $\mathcal{U}$.

Given any object $A \in \mathrm{D}_{\mathcal{U}}^{-}(\mathcal{A})$ there is an integer $N$ such that $\tau_{\leq N} A \rightarrow A$ is a quasiisomorphism where $\tau_{<N} A$ is the intelligent truncation. If we iterate the above construction we find a complex $L$ in $\mathcal{U}$ together with a quasi-isomorphism $L \rightarrow \tau_{\leq N} A$. This shows that for any object $A \in \mathrm{D}_{\mathcal{U}}^{-}(\mathcal{A})$ there is an object $L \in \mathrm{D}^{-}(\mathcal{U})$ together with a quasiisomorphism $L \rightarrow A$. This gives essential surjectivity of our functor and also fully faithfulness by using the description of morphisms by roofs.

Remark 2.3. Assume that the morphisms $U \rightarrow A^{N}$ and $V \rightarrow \mathrm{Z}^{N}(A)$ in the above proof are monomorphisms, i. e. $U \subset A^{N}$ and $V \subset \mathrm{Z}^{N}(A)$. Then one can replace $V \oplus U$ in the above diagram by $V+U \subset A^{N}$ and, proceeding in this way, eventually obtain a quasi-isomorphic subcomplex of $A$ with terms in $\mathcal{U}$.

The full subcategory of projective resp. injective objects in an abelian category $\mathcal{A}$ is denoted below by $\operatorname{proj}(\mathcal{A}) \operatorname{resp} \operatorname{inj}(\mathcal{A})$. We abbreviate $\operatorname{Proj}(R):=\operatorname{proj}(\operatorname{Mod}(R))$ and 
$\operatorname{proj}(R):=\operatorname{proj}(\bmod (R))$ and $\operatorname{inj}(R):=\operatorname{inj}(\bmod (R))$. Note that $\operatorname{proj}(R)=\operatorname{Proj}(R) \cap$ $\bmod (R)$.

We write $\mathrm{K}(\mathcal{A})$ for the homotopy category of an additive category $\mathcal{A}$. Let $\mathcal{A}$ be an abelian category. Then an object $P \in \mathrm{K}(\mathcal{A})$ is called h-projective if $\operatorname{Hom}_{\mathrm{K}(\mathcal{A})}(P, A)=0$ for all acyclic complexes $A \in \mathrm{K}(\mathcal{A})$. For any h-projective complex $P$ and any complex $K \in \mathrm{K}(\mathcal{A})$, the map $\operatorname{Hom}_{\mathrm{K}(\mathcal{A})}(P, K) \rightarrow \operatorname{Hom}_{\mathrm{D}(\mathcal{A})}(P, K)$ induced by the functor $\mathrm{K}(\mathcal{A}) \rightarrow$ $\mathrm{D}(\mathcal{A})$ is an isomorphism. Similarly, an object $I \in \mathrm{K}(\mathcal{A})$ is said to be h-injective if $\operatorname{Hom}_{\mathrm{K}(\mathcal{A})}(A, I)=0$ for all acyclic complexes $A \in \mathrm{K}(\mathcal{A})$. For any h-injective complex $I$ and any complex $K \in \mathrm{K}(\mathcal{A})$, the map $\operatorname{Hom}_{\mathrm{K}(\mathcal{A})}(K, I) \rightarrow \operatorname{Hom}_{\mathrm{D}(\mathcal{A})}(K, I)$ is an isomorphism.

\section{A Negative Result}

Recall that the following conditions on a ring $R$ are equivalent (see [NY03, Theorem 1.50], [Lam99, (15.9) Theorem]):

- $R$ is quasi-Frobenius, i. e. left and right artinian and left and right self-injective;

- $R$ is left or right artinian, and left or right self-injective;

- $R$ is left or right noetherian, and left or right self-injective;

- an arbitrary $R$-module is projective if and only if it is injective.

If $R$ is a quasi-Frobenius ring, any $R$-module has projective dimension either 0 or $\infty$; hence the global dimension of $R$ is either 0 or $\infty$ (note that left and right global dimension of $R$ coincide by [Aus55, Corollary 5]).

Examples of quasi-Frobenius rings are $\mathbb{Z} / n \mathbb{Z}$ for any natural number $n \geq 1$ and $k[\varepsilon] /\left(\varepsilon^{n}\right)$ for any field $k$ and any natural number $n \geq 1$. We first proved the following theorem for the ring $k[\varepsilon] /\left(\varepsilon^{2}\right)$ of dual numbers and advise the reader to keep this example in mind during a first reading of its proof.

Theorem 3.1. Let $R$ be a quasi-Frobenius ring of infinite global dimension. Then the functor

$$
F: \mathrm{D}(\bmod (R)) \rightarrow \mathrm{D}(\operatorname{Mod}(R))
$$

is not full and the closure of its essential image under direct summands in $\mathrm{D}(\operatorname{Mod}(R))$ is strictly contained in $\mathrm{D}_{\bmod (R)}(\operatorname{Mod}(R))$. In particular, the induced functor $\mathrm{D}(\bmod (R)) \rightarrow$ $\mathrm{D}_{\bmod (R)}(\operatorname{Mod}(R))$ is not an equivalence.

Proof. Since $R$ is not semisimple, there is a finitely generated $R$-module $S \in \bmod (R)$ that is not projective (for example a non-projective quotient of $R$ or a non-projective simple subquotient of $R$ ). Since $R$ is an artinian ring, $S$ has a minimal projective resolution

$$
\ldots \rightarrow P_{n+1} \stackrel{d_{n}}{\rightarrow} P_{n} \rightarrow \ldots \rightarrow P_{1} \stackrel{d_{0}}{\rightarrow} P_{0} \rightarrow S \rightarrow 0 .
$$

This just means that the above sequence is exact, that all $P_{n}$ are in $\operatorname{proj}(R)$, and that $P_{0} \rightarrow S$ and all the induced maps $P_{n+1} \rightarrow U_{n}:=\operatorname{im}\left(d_{n}\right)$ are projective covers, for $n \in \mathbb{N}$. Note also that all $P_{n}$ are non-zero because $S$ has infinite projective dimension.

Consider the complexes

$$
\begin{aligned}
& B_{1}=\left(\ldots \rightarrow 0 \rightarrow 0 \rightarrow 0 \rightarrow P_{2} \stackrel{d}{\rightarrow} P_{1} \stackrel{d}{\rightarrow} P_{0} \rightarrow 0 \rightarrow 0 \rightarrow 0 \rightarrow \ldots\right), \\
& B_{2}=\left(\ldots \rightarrow 0 \rightarrow 0 \rightarrow P_{4} \stackrel{d}{\rightarrow} P_{3} \stackrel{d}{\rightarrow} P_{2} \stackrel{d}{\rightarrow} P_{1} \stackrel{d}{\rightarrow} P_{0} \rightarrow 0 \rightarrow 0 \rightarrow \ldots\right), \\
& B_{3}=\left(\ldots \rightarrow 0 \rightarrow P_{6} \stackrel{d}{\rightarrow} P_{5} \stackrel{d}{\rightarrow} P_{4} \stackrel{d}{\rightarrow} P_{3} \stackrel{d}{\rightarrow} P_{2} \stackrel{d}{\rightarrow} P_{1} \stackrel{d}{\rightarrow} P_{0} \rightarrow 0 \rightarrow \ldots\right),
\end{aligned}
$$


in $\operatorname{proj}(R)$ where $B_{n}$ is concentrated in degrees $[-n, n]$ with $P_{n}$ in degree zero. Let

$$
M:=\bigoplus_{n \geq 1} B_{n}
$$

be their coproduct in the abelian category of complexes and also in $\mathrm{D}(\operatorname{Mod}(R))$. The cohomology of $M$ is 0 in degree zero, $S$ in each positive degree and $U_{2 n}$ in degree $-n$, for each $n \geq 1$. Hence $M \in \mathrm{D}_{\bmod (R)}(\operatorname{Mod}(R))$. We claim that $M$ is not in the closure of the essential image of $F$ under direct summands in $\mathrm{D}(\operatorname{Mod}(R))$.

Consider the canonical injective map

$$
\mu: M=\bigoplus_{n \geq 1} B_{n} \rightarrow M^{\prime}:=\prod_{n \geq 1} B_{n}
$$

between the coproduct and the product of the complexes $B_{n}$. The cohomology of $M^{\prime}$ coincides with that of $M$, and $\mu$ is a quasi-isomorphism and becomes an isomorphism in $\mathrm{D}(\operatorname{Mod}(R))$.

Since all projective $R$-modules are injective, all $B_{n}$ are bounded complexes of projective and injective $R$-modules and therefore are h-projective and h-injective (both in $\mathrm{K}(\bmod (R))$ and in $\mathrm{K}(\operatorname{Mod}(R)))$. Since $M$ is also the coproduct of the $B_{n}$ in the homotopy category $\mathrm{K}(\operatorname{Mod}(R))$, it is h-projective. Since $M^{\prime}$ is also the product of the $B_{n}$ in the homotopy category $\mathrm{K}(\operatorname{Mod}(R))$, it is h-injective.

Assume that there is an object $N \in \mathrm{D}(\bmod (R))$ such that $M$ is a direct summand of $F(N)$ in $\mathrm{D}(\operatorname{Mod}(R))$. Then there are morphisms $\iota: M \rightarrow F(N)$ and $\pi: F(N) \rightarrow M$ in $\mathrm{D}(\operatorname{Mod}(R))$ such that $\pi \circ \iota=\mathrm{id}_{M}$. Then

$$
\mu=\mu \circ \pi \circ \iota: M \stackrel{\iota}{\rightarrow} F(N) \stackrel{\mu \circ \pi}{\longrightarrow} M^{\prime} .
$$

Since $M$ is h-projective and $M^{\prime}$ is h-injective, $\iota$ and $\mu \circ \pi$ can be represented by honest morphisms of complexes. The composition of representing morphisms is then a morphism

$$
e: M \rightarrow M^{\prime}
$$

of complexes which becomes equal to $\mu$ in $\mathrm{D}(\operatorname{Mod}(R))$ and has the property that the image $e\left(M^{p}\right)=e^{p}\left(M^{p}\right)$ is finitely generated as an $R$-module, for each $p \in \mathbb{Z}$.

Since $M$ is h-projective (or since $M^{\prime}$ is h-injective) there is a homotopy $h: M \rightarrow M^{\prime}$ (of degree one) between $e$ and $\mu$. In particular, we have

$$
e^{0}-\mu^{0}=d_{M^{\prime}}^{-1} \circ h^{0}+h^{1} \circ d_{M}^{0}
$$

as maps $M^{0} \rightarrow M^{\prime 0}$.

Let $\operatorname{rad}(R)$ be the Jacobson radical of $R$ and let $\operatorname{rad}(T)$ be the radical of an $R$-module $T$. Then $R / \operatorname{rad}(R)$ is a semisimple ring and $\operatorname{rad}(T)=\operatorname{rad}(R) T$ (see [AF92, Proposition 15.16, Corollary 15.18]). We write $\bar{T}:=T / \operatorname{rad}(T)=T / \operatorname{rad}(R) T$ for the quotient by the radical and $\bar{f}: \bar{T} \rightarrow \bar{T}^{\prime}$ for the morphism induced by an $R$-module morphism $f: T \rightarrow T^{\prime}$. With this notation, all the differentials $d_{n}: P_{n+1} \rightarrow P_{n}$ of our minimal projective resolution induce zero morphisms

$$
\bar{d}_{n}=0: \bar{P}_{n+1} \rightarrow \bar{P}_{n}
$$

for each $n \in \mathbb{N}$ because our projective covers become isomorphisms when passing to the respective quotients modulo the radicals (see [ARS97, Proposition I.4.3] for finitely generated modules or more generally [AF92, 27.13]). The equalities

$$
\bar{M}^{0}=\frac{M^{0}}{\operatorname{rad}\left(M^{0}\right)}=\frac{M^{0}}{\operatorname{rad}(R) M^{0}}=\frac{\bigoplus_{n \geq 1} P_{n}}{\bigoplus_{n \geq 1} \operatorname{rad}(R) P_{n}}=\bigoplus_{n \geq 1} \bar{P}_{n}
$$


and similarly for $\bar{M}^{1}$ imply $\bar{d}_{M}^{0}=\bigoplus_{n>1} \bar{d}_{n-1}=0$.

We have $\operatorname{rad}(R) M^{\prime 0}=\operatorname{rad}(R) \prod_{n \geq 1} P_{n}=\prod_{n \geq 1} \operatorname{rad}(R) P_{n}$ because $\operatorname{rad}(R)$ is finitely generated from the right. Therefore we obtain equalities

$$
{\overline{M^{\prime}}}^{0}=\frac{M^{\prime 0}}{\operatorname{rad}\left(M^{\prime 0}\right)}=\frac{M^{\prime 0}}{\operatorname{rad}(R) M^{\prime 0}}=\frac{\prod_{n \geq 1} P_{n}}{\prod_{n \geq 1} \operatorname{rad}(R) P_{n}}=\prod_{n \geq 1} \bar{P}_{n}
$$

and similarly for ${\overline{M^{\prime}}}^{-1}$. We deduce $\bar{d}_{M^{\prime}}^{-1}=\prod_{n \geq 1} \bar{d}_{n}=0$.

Hence (3.1) yields

$$
\bar{e}^{0}=\bar{\mu}^{0}
$$

as maps $\bar{M}^{0} \rightarrow{\overline{M^{\prime}}}^{0}$. The image of $\bar{e}^{0}$ is finitely generated as an $R$-module because $e^{0}\left(M^{0}\right)$ has this property. But the map $\bar{\mu}^{0}: \bar{M}^{0} \rightarrow{\overline{M^{\prime}}}^{0}$ is, under the above identifications, the canonical injective map $\bigoplus_{n \geq 1} \bar{P}_{n} \hookrightarrow \prod_{n \geq 1} \bar{P}_{n}$ whose image is not finitely generated because all $P_{n}$ and hence all $\bar{P}_{n}$ are non-zero. This contradiction proves the claim that $M \in \mathrm{D}_{\bmod (R)}(\operatorname{Mod}(R))$ is not in the closure of the essential image of $F$ under direct summands in $\mathrm{D}(\operatorname{Mod}(R))$.

Let us deduce from this that $F$ is not full. Consider the truncation triangle

$$
\tau_{\leq 0} M \rightarrow M \rightarrow \tau_{\geq 1} M \stackrel{\delta}{\rightarrow} \Sigma \tau_{\leq 0} M
$$

with respect to the standard t-structure. Clearly, $\tau_{\geq 1} M \cong \bigoplus_{n \geq 1} \Sigma^{-n} S=: T_{1}$ and $\tau_{\leq 0} M \cong$ $\bigoplus_{n \geq 1} \Sigma^{n} U_{2 n}=: T_{0}$ are in the essential image of $F$. Assuming that $F$ is full, there is a morphism

$$
\delta^{\prime}: T_{1} \rightarrow \Sigma T_{0}
$$

in $\mathrm{D}(\bmod (R))$ whose image $F\left(\delta^{\prime}\right)$ coincides with $\delta$ modulo the above isomorphisms. Complete $\delta^{\prime}$ to a triangle $T_{0} \rightarrow E \rightarrow T_{1} \stackrel{\delta^{\prime}}{\rightarrow} \Sigma T_{0}$. The image of this triangle under $F$ is isomorphic to the above truncation triangle. In particular $F(E) \cong M$. This contradicts the fact proven above that $M$ is not in the essential image of $F$. Hence $F$ is not full.

We do not know whether the functor in Theorem 3.1 is faithful, not even for $R=$ $\mathbb{C}[\varepsilon] /\left(\varepsilon^{2}\right)$. We conclude this section with several remarks, some of which may help to solve this question.

(a) Let $R$ be a left noetherian ring with the following property:

(*) If $N \hookrightarrow M$ is an arbitrary monomorphism of $R$-modules with $N$ finitely generated, then there is a morphism $M \rightarrow Q$ such that $Q$ is finitely generated and such that the composition $N \hookrightarrow M \rightarrow Q$ is injective.

Then the functor induced by $F$ is an equivalence

$$
\mathrm{D}^{+}(\bmod (R)) \stackrel{\sim}{\rightarrow} \mathrm{D}_{\bmod (R)}^{+}(\operatorname{Mod}(R)) .
$$

To prove this, just dualize the proof of Proposition 2.1.

(b) Any quasi-Frobenius ring $R$ satisfies property ( $\star$ ). Indeed, let $N \hookrightarrow M$ be a monomorphism of $R$-modules with $N$ finitely generated. Since $M$ has an injective hull and any injective $R$-module is projective, there is a monomorphism $M \hookrightarrow$ $\bigoplus_{i \in I} R$ for some index set $I$. Let $J \subset I$ be a finite set such that the image of $N \hookrightarrow M \hookrightarrow \bigoplus_{i \in I} R$ lands in $\bigoplus_{j \in J} R$. Define $U:=M \cap \bigoplus_{i \in I \backslash J} R$. Then $M \rightarrow M / U$ does the job.

(c) Let $R$ be a quasi-Frobenius ring (of infinite global dimension - the case that $R$ is semisimple is boring). Note that we have equivalences (2.1) and (3.2) by the 
previous items (a) and (b). Intelligent truncation and the fact that $F$ is t-exact with respect to the standard t-structures then shows that

$$
F: \operatorname{Hom}_{\mathrm{D}(\bmod (R))}(X, Y) \rightarrow \operatorname{Hom}_{\mathrm{D}(\operatorname{Mod}(R))}(F X, F Y)
$$

is bijective in the following two cases:

- $X \in \mathrm{D}^{-}(\bmod (R))$ and $Y \in \mathrm{D}(\bmod (R))$;

- $X \in \mathrm{D}(\bmod (R))$ and $Y \in \mathrm{D}^{+}(\bmod (R))$.

For bijectivity in the first case it is enough to assume that $R$ is left coherent.

(d) Let $R$ be a quasi-Frobenius ring. If there is a non-zero morphism $f: X \rightarrow Y$ in $\mathrm{D}(\bmod (R))$ that becomes zero in $\mathrm{D}(\operatorname{Mod}(R))$ (i. e. if $F$ is not faithful), then, for all integers $m, n \in \mathbb{Z}$, the morphism $f$ factors as

$$
X \rightarrow \tau_{\geq m} X \stackrel{g}{\rightarrow} \tau_{\leq n} Y \rightarrow Y
$$

in $\mathrm{D}(\bmod (R))$, where $g$ is some (obviously non-zero) morphism that becomes zero in $\mathrm{D}(\operatorname{Mod}(R))$.

Proof. Consider the intelligent truncation triangle

$$
\tau_{<m} X \stackrel{u}{\rightarrow} X \stackrel{v}{\rightarrow} \tau_{\geq m} X \stackrel{d}{\rightarrow} \Sigma \tau_{<m} X .
$$

Applying the functor $\operatorname{Hom}(-, Y)$ to this triangle and $\operatorname{Hom}(-, F Y)$ to its image under $F$ yields the commutative diagram

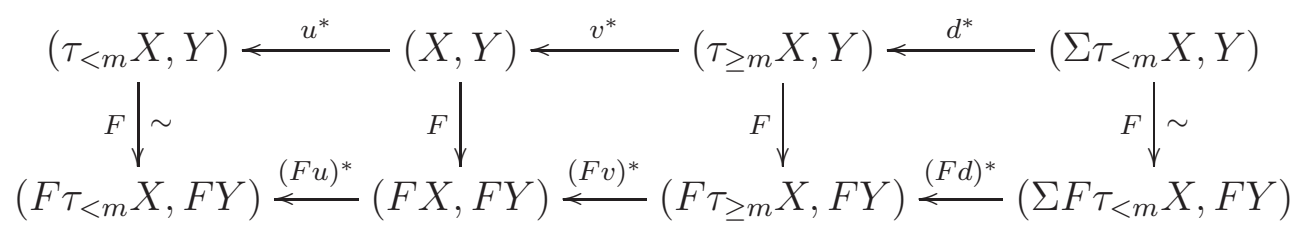

with exact rows. The leftmost and rightmost vertical arrows are bijective by (c). Let $f \in \operatorname{Hom}(X, Y)$ be non-zero with $F(f)=0$. The obvious diagram chase shows that there is some morphism $\hat{f} \in \operatorname{Hom}\left(\tau_{\geq m} X, Y\right)$ with $\hat{f} \circ v=v^{*}(\hat{f})=f$. Then $F(\hat{f})$ goes to zero under $(F v)^{*}$ and another diagram chase yields some morphism $h \in \operatorname{Hom}\left(\Sigma \tau_{<m} X, Y\right)$ such that $F\left(d^{*}(h)\right)=(F(d))^{*}(F(h))=F(\hat{f})$. Hence $F(\hat{f}-h \circ d)$ is zero and the composition $X \stackrel{v}{\rightarrow} \tau_{\geq m} X \stackrel{\hat{f}-h \circ d}{\longrightarrow} Y$ is $f$.

The same argument, now starting with the truncation triangle for $Y$ and the map $\hat{f}-h \circ d$, finishes the proof.

(e) Let $R$ be a quasi-Frobenius ring. Then $\mathrm{D}(\bmod (R))$ is idempotent-complete.

Proof. Let $e: E \rightarrow E$ be an idempotent endomorphism in $\mathrm{D}(\bmod (R))$. Since intelligent truncation is functorial, $\tau_{\leq 0}(e)$ and $\tau_{>0}(e)$ are idempotent endomorphisms. By [LC07, Proposition 2.3] it is enough to show that they split. Since $\bmod (R)$ has enough projectives and injectives (the injective hull of a finitely generated $R$-module is projective and then easily seen to be finitely generated), the obvious functors $\mathrm{K}^{-}(\operatorname{proj}(R)) \rightarrow \mathrm{D}^{-}(\bmod (R))$ and $\mathrm{K}^{+}(\operatorname{inj}(R)) \rightarrow \mathrm{D}^{+}(\bmod (R))$ are equivalences of triangulated categories, where $\mathrm{K}^{-}$resp. $\mathrm{K}^{+}$indicate that only bounded above resp. bounded below complexes are considered. Now note that $\mathrm{K}^{-}(\operatorname{proj}(R))$ and $\mathrm{K}^{+}(\operatorname{inj}(R))$ are idempotent-complete by [Sch11, Theorem 3.1].

\section{First positive Result}

Let $\mathcal{A}$ be an abelian category. The global dimension of $\mathcal{A}$ is the smallest integer $d \geq-1$ with the property that $\operatorname{Ext}_{\mathcal{A}}^{d+1}(M, N)$ vanishes for all objects $M, N \in \mathcal{A}$, if such an integer exists, and $\infty$ otherwise. 
Proposition 4.1. Let $\mathcal{A}$ be an abelian category with enough projective objects that has finite global dimension. Then

(a) for any complex $K$ in $\mathcal{A}$ there is a quasi-isomorphism $P \rightarrow K$ where $P$ has projective components and hence is h-projective by (b);

(b) any complex in $\mathcal{A}$ with projective components is h-projective.

In particular, the obvious functor is an equivalence $\mathrm{K}(\operatorname{proj}(\mathcal{A})) \stackrel{\sim}{\rightarrow} \mathrm{D}(\mathcal{A})$. Hence $\mathrm{D}(\mathcal{A})$ lives in the same universe as $\mathrm{K}(\operatorname{proj}(\mathcal{A}))$.

Proof. Let $d \in \mathbb{N} \cup\{-1\}$ be the global dimension of $\mathcal{A}$. If $d=-1$ then $\mathcal{A}=0$ and all claims are trivial. If $d \geq 0$ we will use the following well-known fact: If $0 \rightarrow L \rightarrow$ $Q_{d-1} \rightarrow \ldots \rightarrow Q_{0} \rightarrow M \rightarrow 0$ is an exact sequence in $\mathcal{A}$ where all $Q_{i}$ are projective, then $L$ is projective. In particular, any object $M$ of $\mathcal{A}$ has a projective resolution of the form $0 \rightarrow Q_{d} \rightarrow Q_{d-1} \rightarrow \ldots \rightarrow Q_{0} \rightarrow M \rightarrow 0$.

(a) Our proof closely follows [Kel98, Appendix]. Let $K$ be a complex in $\mathcal{A}$. Since any object of $M$ has a projective resolution of length $d$, there is a projective resolution

$$
0 \rightarrow P_{d} \rightarrow P_{d-1} \rightarrow \ldots \rightarrow P_{0} \rightarrow K \rightarrow 0
$$

of $K$ in the sense of Cartan-Eilenberg. To construct such a resolution, choose projective resolutions $0 \rightarrow R_{d}^{i} \rightarrow \ldots \rightarrow R_{0}^{i} \rightarrow \mathrm{H}^{i}(K) \rightarrow 0$ and $0 \rightarrow Q_{d}^{i} \rightarrow \ldots \rightarrow Q_{0}^{i} \rightarrow \mathrm{B}^{i}(K) \rightarrow 0$ for all $i \in \mathbb{Z}$ and combine these resolutions of all cohomology and coboundary objects in the obvious way to projective resolutions of all cocycle objects $\mathrm{Z}^{i}(K)$ and then to projective resolutions of all components $K^{i}$.

Let $P$ be the total complex of $0 \rightarrow P_{d} \rightarrow P_{d-1} \rightarrow \ldots \rightarrow P_{0} \rightarrow 0$. Then all components of $P$ are projective and the obvious map $P \rightarrow K$ is clearly a quasi-isomorphism. This proves (a).

We now show that $P$ is h-projective. Each complex $P_{p}$ has the form

$$
\ldots \rightarrow Q_{p}^{i} \oplus R_{p}^{i} \oplus Q_{p}^{i+1} \stackrel{d}{\rightarrow} Q_{p}^{i+1} \oplus R_{p}^{i+1} \oplus Q_{p}^{i+2} \rightarrow \ldots
$$

where the differential $d$ is the composition of the projection to and the inclusion from $Q_{p}^{i+1}$. Hence $P_{p}$ is isomorphic in $\mathrm{K}(\mathcal{A})$ to the complex with vanishing differentials whose component in degree $i$ is the projective object $R_{p}^{i}$; this latter complex is certainly hprojective. Hence $P_{p}$ is h-projective.

If $P_{\leq l}$ denotes the total complex of $0 \rightarrow P_{l} \rightarrow \ldots \rightarrow P_{0} \rightarrow 0$ we have triangles $P_{\leq l} \rightarrow P_{\leq l+1} \rightarrow \Sigma^{l+1} P_{l+1} \rightarrow$ in $\mathrm{K}(\mathcal{A})$ for all $l$ with $0 \leq l \leq d$. Since $P_{0}=P_{\leq 0}$ and all $P_{p}$ are h-projective we see by induction that all $P_{\leq l}$ are h-projective. In particular, $P=P_{\leq d}$ is h-projective.

(b) Let $Q$ be a complex in $\mathcal{A}$ with projective components. We just proved that there is a quasi-isomorphism $f: P \rightarrow Q$ where $P$ has projective components and is h-projective. It is enough to show that $f$ is an isomorphism in $K(\mathcal{A})$. Equivalently, we show that the standard cone $C$ of $f$ is zero in $\mathrm{K}(\mathcal{A})$. Note that $C$ is an acyclic complex with projective components. These two properties will imply that $C$ is isomorphic to 0 in $\mathrm{K}(\mathcal{A})$. Consider the exact sequence

$$
0 \rightarrow \mathrm{Z}^{-d+1}(C) \rightarrow C^{-d+1} \rightarrow \ldots \rightarrow C^{-1} \rightarrow C^{0} \rightarrow \mathrm{Z}^{1}(C) \rightarrow 0
$$

in $\mathcal{A}$ where $\mathrm{Z}^{i}(C)$ is the $i$-th cocycle object. Since all $C^{i}$ are projective and $d$ is the global dimension of $\mathcal{A}$, the cocycle object $\mathrm{Z}^{-d+1}(C)$ is projective. The obvious shift of this argument shows that all cocycle objects $\mathrm{Z}^{i}(C)$ are projective. But then all short exact sequences $\mathrm{Z}^{i}(C) \hookrightarrow C^{i} \rightarrow \mathrm{Z}^{i+1}(C)$ split and it is easy to see that $C$ is contractible, i. e. zero in $\mathrm{K}(\mathcal{A})$. 
This proves (a) and (b). The remaining statements then follow from general properties of h-projective objects explained at the end of section 2 .

Proposition 4.2. Let $\mathcal{A}$ be an abelian category with exact countable coproducts, and let $\mathcal{U} \subset \mathcal{A}$ be a non-empty, full subcategory closed under kernels, cokernels, and extensions. Assume that the abelian category $\mathcal{U}$ has finite global dimension, and that for any epimorphism $A \rightarrow U$ in $\mathcal{A}$ with $U \in \mathcal{U}$ there exists a morphism $V \rightarrow A$ with $V \in \mathcal{U}$ such that the composition $V \rightarrow A \rightarrow U$ is an epimorphism. Then the functor $\mathrm{D}(\mathcal{U}) \rightarrow \mathrm{D}_{\mathcal{U}}(\mathcal{A})$ is essentially surjective.

Proof. Let $M$ be an object of $\mathrm{D}_{\mathcal{U}}(\mathcal{A})$. Consider its intelligent truncation $\tau_{\leq 0} M \in \mathrm{D}_{\mathcal{U}}^{\leq 0}(\mathcal{A})$. Then the proof of Proposition 2.1 shows that there is a complex $L_{0} \in \mathrm{D}^{\leq 0}(\mathcal{U})$ together with a quasi-isomorphism $f_{0}: L_{0} \rightarrow \tau_{\leq 0} M$ of complexes in $\mathcal{A}$.

Let $d \in \mathbb{N} \cup\{-1\}$ be the global dimension of $\mathcal{U}$. We explain the following diagram in $\mathrm{D}(\mathcal{A})$ - some parts come from honest morphisms and inclusions of complexes.

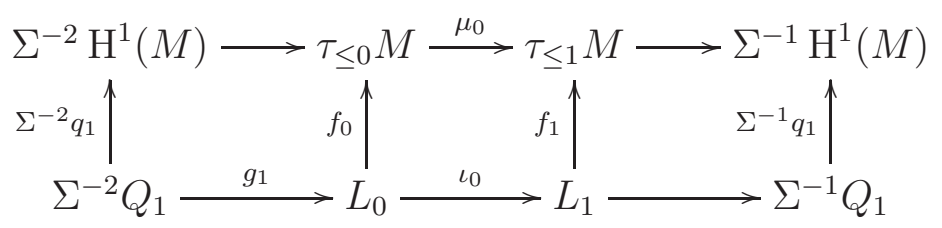

The upper row is a rotation of the obvious truncation triangle in $\mathrm{D}(\mathcal{A})$ where $\mu_{0}$ is the obvious inclusion of complexes. To construct the lower row, recall that $f_{0}: L_{0} \rightarrow \tau_{\leq 0} M$ is an isomorphism in $\mathrm{D}(\mathcal{A})$. Therefore, there exists a morphism $h_{1}: \Sigma^{-2} \mathrm{H}^{1}(M) \rightarrow L_{0}$ in $\mathrm{D}(\mathcal{A})$ such that the map $\Sigma^{-2} \mathrm{H}^{1}(M) \rightarrow \tau_{\leq 0} M$ from the upper row of (4.1) factors as $\Sigma^{-2} \mathrm{H}^{1}(M) \stackrel{h_{1}}{\rightarrow} L_{0} \stackrel{f_{0}}{\rightarrow} \tau_{\leq 0} M$. The morphism $h_{1}$ can be represented by a roof consisting of a quasi-isomorphism $\Sigma^{-2} K_{1} \rightarrow \Sigma^{-2} \mathrm{H}^{1}(M)$ and a morphism of complexes $\Sigma^{-2} K_{1} \rightarrow L_{0}$. Using the argument from the proof of Proposition 2.1, we can assume that $K_{1} \in \mathrm{D}^{\leq 0}(\mathcal{U})$ (since $\mathrm{H}^{1}(M) \in \mathcal{U}$ ).

Our next aim is to show that the complex $K=K_{1}$ can be replaced by a bounded complex with terms in the cohomological degrees from $-d$ to 0 . Indeed, let $\mathrm{Z}^{-d}(K)$ be the image of the differential $K^{-d-1} \rightarrow K^{-d}$. Then the exact sequence $0 \rightarrow \mathrm{Z}^{-d}(K) \rightarrow K^{-d} \rightarrow$ $K^{-d+1} \rightarrow \ldots \rightarrow K^{1} \rightarrow K^{0} \rightarrow \mathrm{H}^{0}(K) \rightarrow 0$ (where $\left.\mathrm{H}^{0}(K) \cong \mathrm{H}^{1}(M)\right)$ represents a Yoneda extension class in the group $\operatorname{Ext}_{\mathcal{U}}^{d+1}\left(\mathrm{H}^{0}(K), \mathrm{Z}^{-d}(K)\right)$. Since $\mathcal{U}$ has global dimension $d$, this Ext group vanishes. By construction of Yoneda Ext, this means that there is a roof connecting our exact sequence with the exact sequence $0 \rightarrow \mathrm{Z}^{-d}(K) \stackrel{\text { id }}{\rightarrow} \mathrm{Z}^{-d}(K) \rightarrow 0 \rightarrow$ $\ldots \rightarrow 0 \rightarrow \mathrm{H}^{0}(K) \stackrel{\text { id }}{\rightarrow} \mathrm{H}^{0}(K) \rightarrow 0$ representing the trivial extension class. So we have the following commutative diagram with exact rows in the category $\mathcal{U}$.

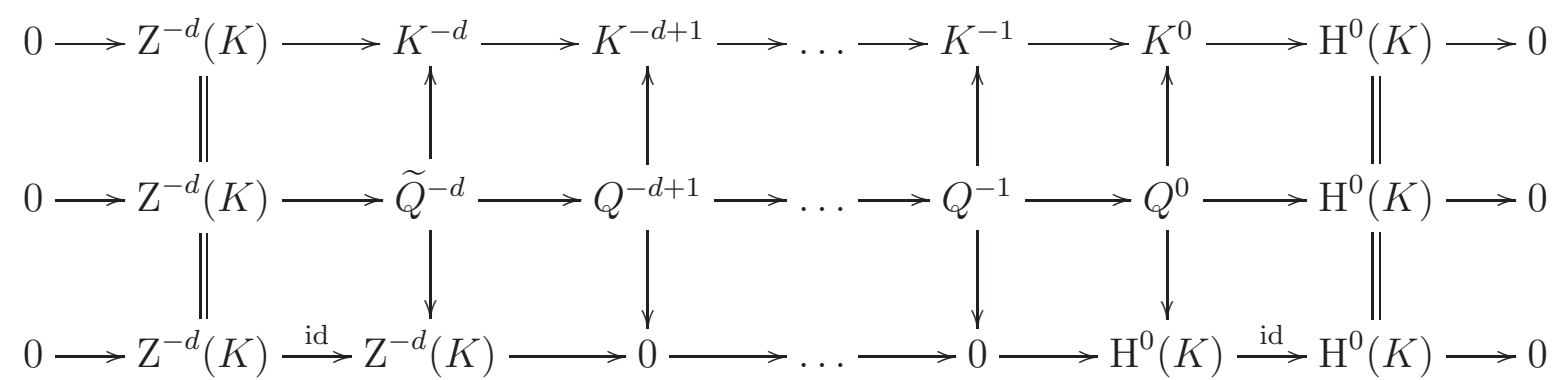

It is clear from this diagram that the monomorphism $\mathrm{Z}^{-d}(K) \rightarrow \widetilde{Q}^{-d}$ splits; so $\widetilde{Q}^{-d} \cong$ $\mathrm{Z}^{-d}(K) \oplus Q^{-d}$ for some object $Q^{-d} \in \mathcal{U}$. We have constructed a bounded complex $Q:=\left(Q^{-d} \rightarrow Q^{-d+1} \rightarrow \ldots \rightarrow Q^{0}\right)$ in $\mathcal{U}$ together with a quasi-isomorphism $Q \rightarrow K$. 
Now we can finish our construction of diagram (4.1). Put $Q_{1}=Q$, and let $q_{1}: Q_{1} \rightarrow$ $\mathrm{H}^{1}(M)$ be the composition $Q_{1} \rightarrow K_{1} \rightarrow \mathrm{H}^{1}(M)$ of quasi-isomorphisms of complexes. Furthermore, let $g_{1}: \Sigma^{-2} Q_{1} \rightarrow L_{0}$ be the composition $\Sigma^{-2} Q_{1} \rightarrow \Sigma^{-2} K_{1} \rightarrow L_{0}$ of morphisms of complexes. Then the leftmost square in $(4.1)$ is commutative in $\mathrm{D}(\mathcal{A})$. We define $L_{1}$ as the standard cone of $g_{1}$; in particular, $L_{0} \stackrel{\iota_{0}}{\rightarrow} L_{1} \rightarrow \Sigma^{-1} Q_{1}$ is a short exact sequence of complexes that splits in each degree; we have $L_{1}=L_{0} \oplus \Sigma^{-1} Q_{1}$ if we ignore the differentials. The lower row of our diagram is then the triangle coming from the obvious standard triangle in $\mathrm{K}(\mathcal{U})$. The morphism $f_{1}$ is a (non-unique) morphism in $\mathrm{D}(\mathcal{A})$ completing the two morphisms $\Sigma^{-2} q_{1}$ and $f_{0}$ to a morphism of triangles. Since $q_{1}$ and $f_{0}$ are isomorphisms, $f_{1}$ is an isomorphism in $\mathrm{D}(\mathcal{A})$.

Next we are going to apply the same construction to the intelligent truncation triangle $\Sigma^{-3} \mathrm{H}^{2}(M) \rightarrow \tau_{\leq 1} M \rightarrow \tau_{\leq 2} M \rightarrow \Sigma^{-2} \mathrm{H}^{2}(M)$ and the morphism $f_{1}: L_{1} \rightarrow \tau_{\leq 1} M$. At this stage, unlike $f_{0}$, the morphism $f_{1}$ only exists in the derived category $\mathrm{D}(\mathcal{\mathcal { A }})$; we do not know whether $f_{1}$ comes from an honest morphism of complexes. But this is not a problem for our construction. Since $f_{1}$ is an isomorphism in $\mathrm{D}(\mathcal{A})$, there exists a morphism $h_{2}: \Sigma^{-3} \mathrm{H}^{2}(M) \rightarrow L_{1}$ in $\mathrm{D}(\mathcal{A})$ such that the map $\Sigma^{-3} \mathrm{H}^{2}(M) \rightarrow \tau_{\leq 1} M$ factors as $\Sigma^{-3} \mathrm{H}^{2}(M) \stackrel{h_{2}}{\longrightarrow} L_{1} \stackrel{f_{1}}{\longrightarrow} \tau_{<1} M$. The morphism $h_{2}$ can be represented by a roof consisting of a quasi-isomorphism $\Sigma^{-3} K_{2} \rightarrow \Sigma^{-3} \mathrm{H}^{2}(M)$ and a morphism of complexes $\Sigma^{-3} K_{2} \rightarrow L_{1}$, etc.

Iterating this construction yields for each $n \in \mathbb{N}$ a diagram

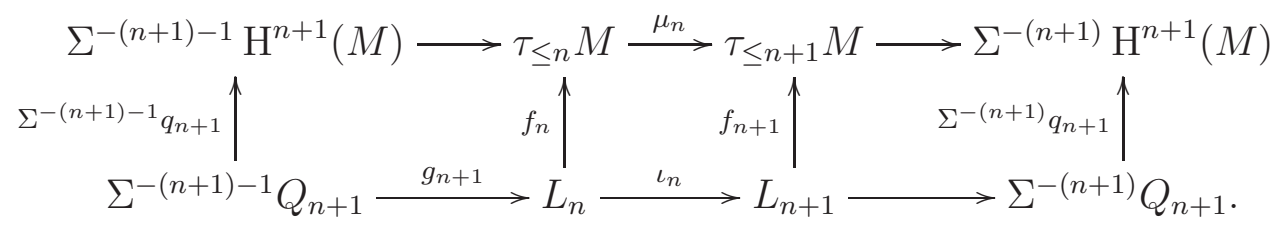

with properties similar to those of (4.1).

Note that $M$ is the colimit of $\tau_{\leq 0} M \subset \tau_{\leq 1} M \subset \ldots$ in the category of complexes in $\mathcal{A}$. Let $L_{\infty}$ be the colimit of $L_{0} \stackrel{\iota_{0}}{\complement} L_{1} \stackrel{\iota_{1}}{\complement} \ldots$ in the category of complexes in $\mathcal{A}$. Then, if we ignore the differentials,

$$
L_{\infty}=L_{0} \oplus \Sigma^{-1} Q_{1} \oplus \Sigma^{-2} Q_{2} \oplus \ldots=L_{0} \oplus \bigoplus_{n \geq 1} \Sigma^{-n} Q_{n}
$$

Since $L_{0}$ and all $Q_{n}$ are complexes in $\mathcal{U}$ and $\Sigma^{-n} Q_{n}$ is concentrated in degrees $[-d+n, n]$, we see that $L_{\infty}$ is a complex in $\mathcal{U}$ as well.

The sequence $\tau_{\leq 0} M \subset \tau_{\leq 1} M \subset \ldots$ of inclusions of complexes is stabilizing in every degree, and the same statement is true for the sequence $L_{0} \stackrel{\iota_{0}}{\complement} L_{1} \stackrel{\iota_{1}}{\complement} \ldots$ of inclusions of complexes (in the latter case all inclusions are even degreewise split). Using the existence of countable coproducts in $\mathcal{A}$, we have degreewise split short exact sequences

$$
\bigoplus_{n \in \mathbb{N}} \tau_{\leq n} M \hookrightarrow \bigoplus_{n \in \mathbb{N}} \tau_{\leq n} M \rightarrow M \quad \text { and } \quad \bigoplus_{n \in \mathbb{N}} L_{n} \hookrightarrow \bigoplus_{n \in \mathbb{N}} L_{n} \rightarrow L_{\infty}
$$

in the abelian category of complexes in $\mathcal{A}$ (where the first map is "identity minus shift" in both cases). These two short exact sequences of complexes give rise to the two horizontal 
triangles in $\mathrm{D}(\mathcal{A})$ in the following diagram.

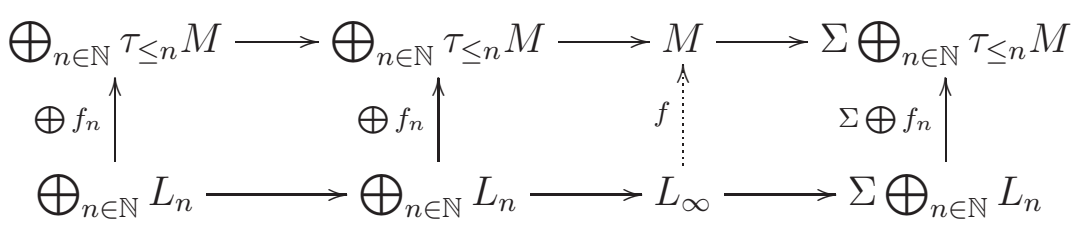

Our assumption that $\mathcal{A}$ has exact countable coproducts implies that countable coproducts in $\mathrm{D}(\mathcal{A})$ exist and can be computed naively. Hence these two triangles exhibit $M$ and $L_{\infty}$ as homotopy colimits. Moreover, taking the coproduct of the morphisms $f_{n}$ in $\mathrm{D}(\mathcal{A})$ defines the vertical morphism $\bigoplus f_{n}$. Note that the square on the left is commutative in $\mathrm{D}(\mathcal{A})$ because $\mu_{n} \circ f_{n}=f_{n+1} \circ \iota_{n}$ for all $n \in \mathbb{N}$. Hence a dotted morphism $f$ exists (non-uniquely) completing this square to a morphism of triangles. This morphism $f$ is an isomorphism because all $f_{n}$ and hence their coproduct $\bigoplus f_{n}$ are isomorphisms. Since $L_{\infty}$ is a complex in $\mathcal{U}$ we deduce that $M$ is in the essential image of our functor $\mathrm{D}(\mathcal{U}) \rightarrow \mathrm{D}_{\mathcal{U}}(\mathcal{A})$

Theorem 4.3. Let $\mathcal{A}$ be an abelian category of finite global dimension with exact countable coproducts, and let $\mathcal{U} \subset \mathcal{A}$ be a non-empty, full subcategory closed under kernels, cokernels, and extensions. Assume that both abelian categories $\mathcal{A}$ and $\mathcal{U}$ have enough projective objects and that $\operatorname{proj}(\mathcal{U}) \subset \operatorname{proj}(\mathcal{A})$ (and hence $\operatorname{proj}(\mathcal{U})=\mathcal{U} \cap \operatorname{proj}(\mathcal{A})$ ). Then the obvious functor is an equivalence $\mathrm{D}(\mathcal{U}) \stackrel{\sim}{\rightarrow} \mathrm{D}_{\mathcal{U}}(\mathcal{A})$ of triangulated categories.

Remark 4.4. If $\mathcal{A}$ is hereditary, i. e. of global dimension $\leq 1$, Theorem 4.3 can be strengthened to Corollary A.2. Neither the assumption that countable coproducts exist nor the assumptions on projective objects are needed in this case.

Proof. Since there are enough projectives in $\mathcal{U}$ and they are also projective in $\mathcal{A}$, it is clear that the inclusion $\mathcal{U} \rightarrow \mathcal{A}$ induces isomorphisms on all Ext groups. Therefore, the global dimension of $\mathcal{U}$ cannot exceed the global dimension of $\mathcal{A}$.

The inclusion $\mathcal{U} \rightarrow \mathcal{A}$ restricts to a fully faithful functor $\operatorname{proj}(\mathcal{U}) \rightarrow \operatorname{proj}(\mathcal{A})$. Hence the induced triangulated functor $\mathrm{K}(\operatorname{proj}(\mathcal{U})) \rightarrow \mathrm{K}(\operatorname{proj}(\mathcal{A}))$ is fully faithful.

Since the category $\mathcal{A}$ has finite global dimension by assumption, Proposition 4.1 can be applied both to $\mathcal{U}$ and $\mathcal{A}$. Hence, the vertical functors in the obvious commutative diagram

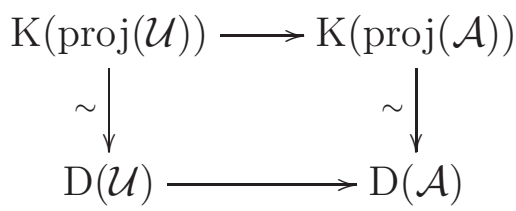

are equivalences. Since the upper horizontal functor is fully faithful, the lower horizontal functor has the same property. It clearly lands in $\mathrm{D}_{\mathcal{U}}(\mathcal{A})$. So it remains to be shown that its essential image is $\mathrm{D}_{\mathcal{U}}(\mathcal{A})$.

Let $A \rightarrow U$ be an epimorphism in $\mathcal{A}$ with $U \in \mathcal{U}$. Choose a projective object $V \in$ $\operatorname{proj}(\mathcal{U})$ together with an epimorphism $V \rightarrow U$. Since $V$ is also projective in $\mathcal{A}$, the epimorphism $V \rightarrow U$ factors through the epimorphism $A \rightarrow U$.

Therefore, Proposition 4.2 is applicable, and we are done.

Corollary 4.5. Let $R$ be a left coherent ring of finite left global dimension. Then the obvious triangulated functor is an equivalence

$$
\mathrm{D}(\bmod (R)) \stackrel{\sim}{\rightarrow} \mathrm{D}_{\bmod (R)}(\operatorname{Mod}(R)) .
$$


Proof. This is a particular case of Theorem 4.3. Note that $\bmod (R)$ has enough projective objects since $R \in \bmod (R)$.

\section{SeCond POsitive RESUlt}

Let $\mathcal{A}$ be an abelian category. Any short exact sequence $0 \rightarrow K \rightarrow L \rightarrow M \rightarrow 0$ of complexes in $\mathcal{A}$ can be considered as a bicomplex with three rows.

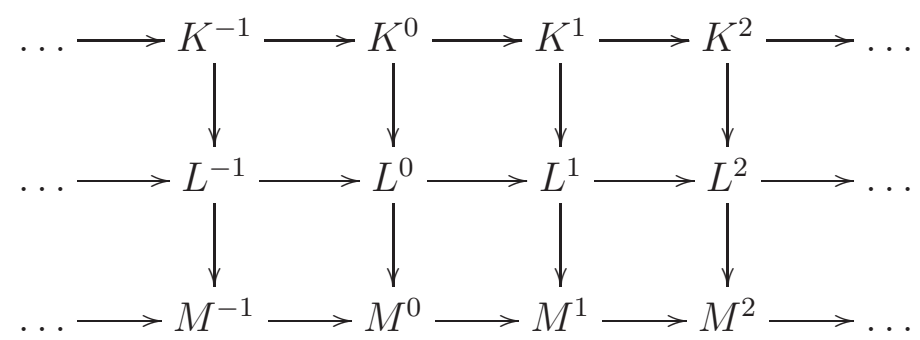

We consider the total complex of this bicomplex and denote it by $\operatorname{Tot}(K \rightarrow L \rightarrow M)$.

A complex in $\mathcal{A}$ is said to be absolutely acyclic if it belongs to the minimal thick subcategory of $\mathrm{K}(\mathcal{A})$ containing all the total complexes $\operatorname{Tot}(K \rightarrow L \rightarrow M)$ of short exact sequences $0 \rightarrow K \rightarrow L \rightarrow M \rightarrow 0$ of complexes in $\mathcal{A}$. Clearly, any absolutely acyclic complex is acyclic, but the converse is not true in general. One can easily see that any bounded acyclic complex is absolutely acyclic.

Example 5.1. The unbounded acyclic complex

$$
\ldots \stackrel{\varepsilon}{\rightarrow} R \stackrel{\varepsilon}{\rightarrow} R \stackrel{\varepsilon}{\rightarrow} R \stackrel{\varepsilon}{\rightarrow} \ldots
$$

of projective-injective modules over the ring of dual numbers $R=k[\varepsilon] /\left(\varepsilon^{2}\right)$ (over any field $k$ ) is not absolutely acyclic. Moreover, both intelligent truncations

$$
\ldots \stackrel{\varepsilon}{\rightarrow} R \stackrel{\varepsilon}{\rightarrow} R \stackrel{\varepsilon}{\rightarrow} R \rightarrow k \rightarrow 0 \quad \text { and } \quad 0 \rightarrow k \hookrightarrow R \stackrel{\varepsilon}{\rightarrow} R \stackrel{\varepsilon}{\rightarrow} R \stackrel{\varepsilon}{\rightarrow} \ldots
$$

of this complex are acyclic, but not absolutely acyclic. Similarly, the unbounded acyclic complex

$$
\ldots \stackrel{2}{\rightarrow} R \stackrel{2}{\rightarrow} R \stackrel{2}{\rightarrow} R \stackrel{2}{\rightarrow} \ldots
$$

of projective-injective modules over the ring $R=\mathbb{Z} / 4 \mathbb{Z}$ is not absolutely acyclic. The intelligent truncations

$$
\ldots \stackrel{2}{\rightarrow} R \stackrel{2}{\rightarrow} R \stackrel{2}{\rightarrow} R \rightarrow \mathbb{Z} / 2 \mathbb{Z} \rightarrow 0 \quad \text { and } \quad 0 \rightarrow \mathbb{Z} / 2 \mathbb{Z} \hookrightarrow R \stackrel{2}{\rightarrow} R \stackrel{2}{\rightarrow} R \stackrel{2}{\rightarrow} \ldots
$$

of this complex are again acyclic, but not absolutely acyclic.

More generally, let $R$ be a quasi-Frobenius ring and let $M$ be a non-projective (and hence non-injective) $R$-module. Let $\ldots \rightarrow P_{2} \rightarrow P_{1} \rightarrow P_{0} \stackrel{\pi}{\rightarrow} M \rightarrow 0$ be a projective resolution of $M$ and let $0 \rightarrow M \stackrel{\iota}{\hookrightarrow} J^{0} \rightarrow J^{1} \rightarrow J^{2} \rightarrow \ldots$ be an injective resolution of $M$. Let $f$ be the composition $P_{0} \stackrel{\pi}{\rightarrow} M \stackrel{\iota}{\hookrightarrow} J^{0}$. Then the three acyclic complexes

$$
\begin{aligned}
\ldots \rightarrow P_{2} & \rightarrow P_{1} \rightarrow P_{0} \stackrel{f}{\rightarrow} J^{0} \rightarrow J^{1} \rightarrow J^{2} \rightarrow \ldots, \\
\ldots \rightarrow P_{2} \rightarrow P_{1} & \rightarrow P_{0} \stackrel{\pi}{\rightarrow} M \rightarrow 0, \\
0 & \rightarrow M \stackrel{\iota}{\rightarrow} J^{0} \rightarrow J^{1} \rightarrow J^{2} \rightarrow \ldots .
\end{aligned}
$$

of $R$-modules are not absolutely acyclic.

To prove these assertions, one can use the notions of coacyclic and contraacyclic complexes. Given an abelian category $\mathcal{A}$ with exact coproducts, one says that a complex in $\mathcal{A}$ is coacyclic if it belongs to the minimal triangulated subcategory of $\mathrm{K}(\mathcal{A})$ containing the 
absolutely acyclic complexes and closed under coproducts. Dually, if an abelian category $\mathcal{A}$ has exact products, a complex in $\mathcal{A}$ is said to be contraacyclic if it belongs to the minimal triangulated subcategory of $K(\mathcal{A})$ containing the absolutely acyclic complexes and closed under products. The following lemmas summarize some key observations.

Lemma 5.2. (a) Let $I \in \mathrm{K}(\operatorname{inj}(\mathcal{A})) \subset \mathrm{K}(\mathcal{A})$ be a complex of injective objects in an abelian category $\mathcal{A}$ with exact coproducts. Then $I$ is coacyclic if and only if it is contractible.

(b) Let $P \in \mathrm{K}(\operatorname{proj}(\mathcal{A})) \subset \mathrm{K}(\mathcal{A})$ be a complex of projective objects in an abelian category $\mathcal{A}$ with exact products. Then $P$ is contraacyclic if and only if it is contractible.

Proof. Being contractible means being zero in $\mathrm{K}(\mathcal{A})$. Hence any contractible complex is coacyclic resp. contraacyclic. The reverse implications follow from [Pos11, Section 3.5] (or [Pos17, Lemma A.1.3]): In part (a), we have $\operatorname{Hom}_{\mathrm{K}(\mathcal{A})}(A, I)=0$ for all coacyclic complexes $A$ in $\mathcal{A}$; if $I$ is coacyclic, take $A=I$. In part (b), we have $\operatorname{Hom}_{\mathrm{K}(\mathcal{A})}(P, B)=0$ for all contraacyclic complexes $B$ in $\mathcal{A}$; if $P$ is contraacyclic, take $B=P$.

Lemma 5.3. (a) Any bounded below acyclic complex in $\mathcal{A}$ is coacyclic.

(b) Any bounded above acyclic complex in $\mathcal{A}$ is contraacyclic.

Proof. This is a particular case of [Pos10, Lemmas in sections 2.1 and 4.1] (see also [Pos11, Theorem 1(a) in section 3.4]).

Now we can show that the three acyclic complexes (5.1), (5.2), (5.3) are not absolutely acyclic. Convince yourself that, given any contractible complex $A$ in an abelian category, all monomorphisms $\mathrm{B}^{n}(A) \hookrightarrow A^{n}$ and all epimorphisms $A^{n-1} \rightarrow \mathrm{B}^{n}(A)$ split. Since $M$ is not projective, $P_{0} \stackrel{\pi}{\rightarrow} M$ does not split (similarly, $M \stackrel{\iota}{\hookrightarrow} J^{0}$ does not split since $M$ is not injective). Hence the complex (5.1) is not contractible. Since all its terms are both projective and injective, Lemma 5.2 shows that it is neither coacyclic nor contraacyclic.

Consider the obvious map $(5.2) \rightarrow(5.1)$ of complexes. Its mapping cone is clearly homotopic to (5.3). Hence there is a triangle $(5.2) \rightarrow(5.1) \rightarrow(5.3) \rightarrow \Sigma(5.2)$ in $\mathrm{K}(\mathcal{A})$. By Lemma 5.3(a), the complex (5.3) is coacyclic. Since the complex (5.1) is not coacyclic, the complex (5.2) cannot be coacyclic. Dually, by Lemma 5.3(b), the complex (5.2) is contraacyclic. Since the complex (5.1) is not contraacyclic, the complex (5.3) cannot be contraacyclic.

By definition, all absolutely acyclic complexes are both coacyclic and contraacyclic. Thus none of the complexes (5.1), (5.2), (5.3) is absolutely acyclic.

Denote the thick subcategory of absolutely acyclic complexes by $\operatorname{Ac}^{\text {abs }}(\mathcal{A}) \subset \mathrm{K}(\mathcal{A})$. The triangulated Verdier quotient category

$$
\mathrm{D}^{\mathrm{abs}}(\mathcal{A})=\mathrm{K}(\mathcal{A}) / \operatorname{Ac}^{\mathrm{abs}}(\mathcal{A})
$$

is called the absolute derived category of the abelian category $\mathcal{A}$.

Since all absolutely acyclic complexes are acyclic, we have a canonical (triangulated Verdier quotient) functor

$$
\mathrm{D}^{\mathrm{abs}}(\mathcal{A}) \rightarrow \mathrm{D}(\mathcal{A}) .
$$

In fact, we obtain a commutative triangular diagram of triangulated Verdier quotient functors $\mathrm{K}(\mathcal{A}) \rightarrow \mathrm{D}^{\text {abs }}(\mathcal{A}) \rightarrow \mathrm{D}(\mathcal{A})$. 
Remark 5.4. We have already mentioned that any bounded acyclic complex is absolutely acyclic. In an abelian category of finite global dimension, any acyclic complex is absolutely acyclic [Pos10, Remark in section 2.1]. This means that the canonical functor $\mathrm{D}^{\mathrm{abs}}(\mathcal{A}) \rightarrow \mathrm{D}(\mathcal{A})$ is an equivalence whenever $\mathcal{A}$ has finite global dimension.

Theorem 5.5. Let $\mathcal{A}$ be an abelian category and let $\mathcal{U} \subset \mathcal{A}$ be a non-empty, full subcategory closed under subobjects, quotients, and extensions. Assume that for any epimorphism $A \rightarrow U$ in $\mathcal{A}$ with $U \in \mathcal{U}$ there exists a subobject $V \subset A$ with $V \in \mathcal{U}$ such that the composition $V \hookrightarrow A \rightarrow U$ is an epimorphism. Then the obvious triangulated functor

$$
\mathrm{D}^{\mathrm{abs}}(\mathcal{U}) \rightarrow \mathrm{D}^{\mathrm{abs}}(\mathcal{A})
$$

is fully faithful.

Remark 5.6. We do not know how to describe the essential image of this functor in a nice way. In general, it is not true that all complexes in $\mathcal{A}$ whose cohomology objects belong to $\mathcal{U}$ are in the essential image: Let $\mathcal{A}$ be an abelian category such that there are acyclic complexes that are not absolutely acyclic (cf. Example 5.1) and consider the subcategory $\mathcal{U}=0$.

Proof. We introduce some additional terminology in order to formulate the argument. First of all, the objects of $\mathrm{Ac}^{\text {abs }}(\mathcal{U})$ will be called absolutely acyclic complexes with respect to $\mathcal{U}$, the objects of $\operatorname{Ac}^{\text {abs }}(\mathcal{A})$ will be called absolutely acyclic complexes with respect to $\mathcal{A}$. Clearly, any absolutely acyclic complex with respect to $\mathcal{U}$ is also absolutely acyclic with respect to $\mathcal{A}$. Conversely, it follows from the assertion of the theorem that a complex in $\mathcal{U}$ that is absolutely acyclic with respect to $\mathcal{A}$ is already absolutely acyclic with respect to $\mathcal{U}$. So $\operatorname{Ac}^{\text {abs }}(\mathcal{U})=\operatorname{Ac}^{\text {abs }}(\mathcal{A}) \cap K(\mathcal{U})$. But this is not obvious and needs to be proved.

We will show that any morphism in $\mathrm{K}(\mathcal{A})$ from a complex in $\mathcal{U}$ to a complex absolutely acyclic with respect to $\mathcal{A}$ factors through a complex absolutely acyclic with respect to $\mathcal{U}$. This is clearly sufficient to prove the theorem (see e.g. [LS16, Proposition B.2(II)]).

The full subcategory $\operatorname{Ac}^{\text {abs }}(\mathcal{A})$ of absolutely acyclic complexes in $K(\mathcal{A})$ is defined by a generation procedure, and the argument proceeds along the steps of this procedure. Let us describe this procedure in more detail. We start with the empty subcategory of $K(\mathcal{A})$ and successively add objects.

Step 1. All total complexes $\operatorname{Tot}(K \rightarrow L \rightarrow M)$ of short exact sequences $0 \rightarrow K \rightarrow L \rightarrow$ $M \rightarrow 0$ of complexes in $\mathcal{A}$ are added.

Step 2. If two complexes $A$ and $B$ have been added in the previous steps, and if $t: A \rightarrow B$ is a morphism of complexes, then we add the complex $C=\operatorname{Cone}(t)$. Let us emphasize that Cone $(t)$ is understood here as the cone of a closed morphism of complexes; so there exists a short exact sequence $0 \rightarrow B \rightarrow C \rightarrow \Sigma(A) \rightarrow 0$ in the abelian category of complexes in $\mathcal{A}$. All the complexes $C$ that can be obtained in this way are added in this step.

Step 3. Step 2 is repeated countably many times.

Step 4. If a complex $A$ has been added already and a complex $B$ is homotopy equivalent to $A$, then the complex $B$ is added in this step.

Step 5. If a complex $A$ has been added already and a complex $B$ is a direct summand of $A$ in $\mathrm{K}(\mathcal{A})$, then the complex $B$ is added in this step.

After all the steps 1-5 have been run, we have precisely produced the full subcategory $\operatorname{Ac}^{\text {abs }}(\mathcal{A}) \subset \mathrm{K}(\mathcal{A})$.

Let us say that a complex $A \in \mathrm{K}(\mathcal{A})$ is approachable if any morphism $f: U \rightarrow A$ in $\mathrm{K}(\mathcal{A})$ with $U \in \mathrm{K}(\mathcal{U})$ factors through a complex absolutely acyclic with respect to $\mathcal{U}$. 
Moreover, we will say that a complex $A$ is strongly approachable if, for any subcomplex $D \subset A$ with terms $D^{n} \in \mathcal{U}, n \in \mathbb{Z}$, there exists a subcomplex $E \subset A$ such that $D \subset E$ and the complex $E$ is absolutely acyclic with respect to $\mathcal{U}$. Since $\mathcal{U}$ is closed under quotients, any strongly approachable complex is approachable. Our aim is to show that all complexes $A \in \operatorname{Ac}^{\text {abs }}(\mathcal{A})$ are approachable.

Approachability is a concept defined on the level of homotopy categories. Strong approachability is a concept defined on the level of abelian categories of complexes. In the above generation procedure, steps 1-3 take place on the abelian level. Steps 4-5 take place on the level of the homotopy category.

The proof of the theorem now proceeds as a sequence of lemmas.

Lemma 5.7. For any short exact sequence $0 \rightarrow K \rightarrow L \rightarrow M \rightarrow 0$ of complexes in $\mathcal{A}$, the complex $A=\operatorname{Tot}(K \rightarrow L \rightarrow M)$ is strongly approachable.

Proof. We have $A^{n}=K^{n+1} \oplus L^{n} \oplus M^{n-1}$ for all $n \in \mathbb{Z}$. Let $D \subset A$ be a subcomplex with terms $D^{n} \in \mathcal{U}$. Denote by $D_{K}^{n+1} \subset K^{n+1}, D_{L}^{n} \subset L^{n}$, and $D_{M}^{n-1} \subset M^{n-1}$ the images of the projections of the subobject $D^{n} \subset A^{n}$ onto the three direct summands of the object $A^{n}$. So we have $D^{n} \subset D_{K}^{n+1} \oplus D_{L}^{n} \oplus D_{M}^{n-1}$ and all three objects $D_{K}^{n+1}, D_{L}^{n}, D_{M}^{n-1}$ belong to $\mathcal{U}$.

By assumption, for each $n \in \mathbb{Z}$ we can choose a subobject $G^{n} \subset L^{n}$ such that $G^{n} \in$ $\mathcal{U}$ and such that the image of $G^{n}$ under the epimorphism $L^{n} \rightarrow M^{n}$ coincides with $D_{M}^{n} \subset M^{n}$. Denote by $H^{n} \subset L^{n}$ the image of the composition of monomorphisms $D_{K}^{n} \hookrightarrow K^{n} \hookrightarrow L^{n}$. Put $Q^{n}=H^{n}+D_{L}^{n}+G^{n} \subset L^{n}$ and $V^{n}=Q^{n}+d\left(Q^{n-1}\right) \subset L^{n}$. Clearly, we have $Q^{n} \in \mathcal{U}$ and consequently $V^{n} \in \mathcal{U}$.

Now $V$ is a subcomplex of $L$. Denote by $U$ the preimage of $V$ under the monomorphism of complexes $K \hookrightarrow L$, and by $W$ the image of $V$ under the epimorphism of complexes $L \rightarrow M$. Then we have a short exact sequence $0 \rightarrow U \rightarrow V \rightarrow W \rightarrow 0$ of complexes in $\mathcal{U}$. The complex $E=\operatorname{Tot}(U \rightarrow V \rightarrow W)$ is absolutely acyclic with respect to $\mathcal{U}$, and it is a subcomplex in $A$. Finally, we have $D_{K}^{n} \subset U^{n} \subset K^{n}, D_{L}^{n} \subset V^{n} \subset L^{n}$, and $D_{M}^{n} \subset W^{n} \subset M^{n}$, hence $D \subset E \subset A$, as desired.

Lemma 5.8. If $t: A \rightarrow B$ is a morphism of complexes in $\mathcal{A}$ and the two complexes $A$ and $B$ are strongly approachable, then the complex $C=$ Cone $(t)$ is strongly approachable.

Proof. The terms of the complex $C$ are $C^{n}=A^{n+1} \oplus B^{n}$. Let $D \subset C$ be a subcomplex with terms $D^{n} \in \mathcal{U}$. Denote by $D_{A}^{n+1} \subset A^{n+1}$ and $D_{B}^{n} \subset B^{n}$ the images of the projections of the subobject $D^{n} \subset C^{n}$ onto the two direct summands of $C^{n}$. So we have $D^{n} \subset D_{A}^{n+1} \oplus D_{B}^{n}$ and $D_{A}^{n+1}, D_{B}^{n} \in \mathcal{U}$.

Put $P^{n}=D_{A}^{n}+d\left(D_{A}^{n-1}\right) \subset A^{n}$. Then $P$ is a subcomplex of $A$ with terms $P^{n} \in \mathcal{U}$. By assumption, there exists a subcomplex $E \subset A$ such that $E$ is absolutely acyclic with respect to $\mathcal{U}$ and $P \subset E \subset A$. In particular, we have $E^{n} \in \mathcal{U}$ for all $n \in \mathbb{Z}$. Put $Q^{n}=D_{B}^{n}+d\left(D_{B}^{n-1}\right)+t\left(E^{n}\right) \subset B^{n}$. Then $Q$ is a subcomplex of $B$ with terms $Q^{n} \in \mathcal{U}$. By assumption, there exists a subcomplex $F \subset B$ such that $F$ is absolutely acyclic with respect to $\mathcal{U}$ and $Q \subset F \subset B$.

By construction, we have $t(E) \subset F$; denote by $h: E \rightarrow F$ the morphism induced by $t$. Put $G=$ Cone $(h)$. Then the complex $G$ is absolutely acyclic with respect to $\mathcal{U}$, and it is a subcomplex of $C$. Finally, we have $D_{A}^{n} \subset E^{n} \subset A^{n}$ and $D_{B}^{n} \subset F^{n} \subset B^{n}$, hence $D \subset G \subset C$, as desired.

Lemma 5.9. (a) If a complex $A$ is approachable and a complex $B$ is homotopy equivalent to $A$, then the complex $B$ is approachable as well.

(b) If a complex $A \in \mathrm{K}(\mathcal{A})$ is approachable and a complex $B$ is a direct summand of $A$ in $\mathrm{K}(\mathcal{A})$, then the complex $B$ is approachable as well. 
Proof. This follows immediately from the definitions.

It remains to finish the proof of the theorem. All the complexes produced in step 1 are strongly approachable by Lemma 5.7. Hence all the complexes produced in steps 2 and 3 are strongly approachable by Lemma 5.8. It follows that all complexes produced in steps 4 and 5 are approachable by Lemma 5.9. Thus all complexes that are absolutely acyclic with respect to $\mathcal{A}$ are approachable.

Theorem 5.10. Let $\mathcal{A}$ be an abelian category of finite global dimension with exact countable coproducts, and let $\mathcal{U} \subset \mathcal{A}$ be a non-empty, full subcategory closed under subobjects, quotients, and extensions. Assume that for any epimorphism $A \rightarrow U$ in $\mathcal{A}$ with $U \in \mathcal{U}$ there exists a subobject $V \subset A$ with $V \in \mathcal{U}$ such that the composition $V \hookrightarrow A \rightarrow U$ is an epimorphism. Then the obvious triangulated functor is an equivalence $\mathrm{D}(\mathcal{U}) \stackrel{\sim}{\rightarrow} \mathrm{D}_{\mathcal{U}}(\mathcal{A})$.

Proof. Proposition 2.1 shows that the functor $\mathrm{D}^{-}(\mathcal{U}) \rightarrow \mathrm{D}_{\mathcal{U}}^{-}(\mathcal{A})$ is an equivalence of triangulated categories. In particular, the inclusion of abelian categories $\mathcal{U} \rightarrow \mathcal{A}$ induces isomorphisms on all Ext groups. Therefore, the global dimension of $\mathcal{U}$ cannot exceed the global dimension of $\mathcal{A}$.

Since the global dimensions of the abelian categories $\mathcal{U}$ and $\mathcal{A}$ are finite, both vertical functors in the obvious commutative diagram

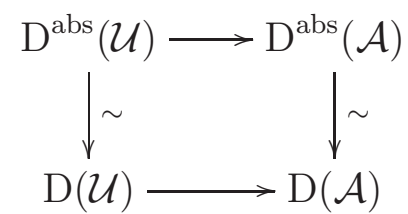

of triangulated functors are equivalences by Remark 5.4. The upper horizontal functor is fully faithful by Theorem 5.5. It follows that the lower horizontal functor is fully faithful. Finally, the functor $\mathrm{D}(\mathcal{U}) \rightarrow \mathrm{D}_{\mathcal{U}}(\mathcal{A})$ is essentially surjective by Proposition 4.2.

The following corollary concerns locally noetherian Grothendieck abelian categories (see [Gab62, Section II.4]).

Corollary 5.11. Let $\mathcal{A}$ be a locally noetherian Grothendieck category of finite global dimension and let $\mathcal{U} \subset \mathcal{A}$ be the full subcategory of noetherian objects. Then the obvious triangulated functor is an equivalence $\mathrm{D}(\mathcal{U}) \stackrel{\sim}{\rightarrow} \mathrm{D}_{\mathcal{U}}(\mathcal{A})$.

Proof. This is a particular case of Theorem 5.10 because the categories $\mathcal{A}$ and $\mathcal{U}$ clearly satisfy the necessary assumptions.

For a noetherian scheme $X$, we denote by $\mathrm{Q} \operatorname{coh}(X)$ the abelian category of quasicoherent sheaves on $X$ and by $\operatorname{coh}(X) \subset \mathrm{Q} \operatorname{coh}(X)$ the full subcategory of coherent sheaves. The notation $\mathrm{D}_{\text {coh }}(\mathrm{Q} \operatorname{coh}(X))$ stands for the full triangulated subcategory of $\mathrm{D}(\mathrm{Q} \operatorname{coh}(X))$ consisting of all complexes with coherent cohomology sheaves.

Corollary 5.12. Let $X$ be a regular noetherian scheme of finite Krull dimension. Then the obvious triangulated functor is an equivalence

$$
\mathrm{D}(\operatorname{coh}(X)) \stackrel{\sim}{\rightarrow} D_{\operatorname{coh}}(\mathrm{Q} \operatorname{coh}(X)) .
$$

Proof. For any noetherian scheme $X$, the category $Q \operatorname{coh}(X)$ is locally noetherian [Gab62, Théorème VI.2.1] and its subcategory of noetherian objects is $\operatorname{coh}(X)$. When $X$ is regular of Krull dimension $d$, the global dimension of $\mathrm{Qcoh}(X)$ is equal to $d$. This well-known result is provable as follows.

Assume first that $X=\operatorname{Spec} R$ is affine. Let $M$ be a finitely generated $R$-module. Let $0 \rightarrow Z \rightarrow P_{d-1} \rightarrow \ldots \rightarrow P_{0} \rightarrow M \rightarrow 0$ be an exact sequence where all $P_{i}$ are finitely 
generated projective $R$-modules. If we localize this sequence at an arbitrary prime ideal $\mathfrak{p} \in$ Spec $R$, the $R_{\mathfrak{p}}$-module $Z_{\mathfrak{p}}$ is projective because the regular noetherian local ring $R_{\mathfrak{p}}$ has global dimension equal to $\operatorname{dim} R_{\mathfrak{p}} \leq \operatorname{dim} R=d$ by [Mat89, Theorem 19.2]. Hence $Z$ is projective as an $R$-module. This shows that $\bmod (R) \cong \operatorname{coh}(\operatorname{Spec} R)$ has global dimension $\leq d$. But this implies that $\operatorname{Mod}(R) \cong \mathrm{Q} \operatorname{coh}(\operatorname{Spec} R)$ has global dimension $\leq d$ and in fact equal to $d$ by [Rot09, Theorem 8.16, Theorem 8.55].

Now assume that $X$ is not necessarily affine. By [Har66, Theorem II.7.18], any $\mathcal{F} \in$ Qcoh $(X)$ admits an embedding $\mathcal{F} \subset \mathcal{I}$ where $\mathcal{I}$ is quasi-coherent and injective as an $\mathcal{O}_{X^{-}}$ module and hence in particular injective as an object of $Q \operatorname{coh}(X)$. Since this embedding splits if $\mathcal{F}$ is injective in $\operatorname{Qcoh}(X)$, we obtain $\operatorname{inj}(\operatorname{Qcoh}(X))=\operatorname{inj}\left(\operatorname{Mod}\left(\mathcal{O}_{X}\right)\right) \cap \operatorname{Qcoh}(X)$. From [Har66, Lemma II.7.16] we see that an object of $\mathrm{Q} \operatorname{coh}(X)$ is injective if and only if its restrictions to an arbitrary open cover of $X$ are injective as quasi-coherent sheaves.

Given $\mathcal{F} \in \operatorname{Qcoh}(X)$, let $0 \rightarrow \mathcal{F} \rightarrow \mathcal{I}_{0} \rightarrow \ldots \rightarrow \mathcal{I}_{d-1} \rightarrow \mathcal{C} \rightarrow 0$ be an exact sequence in $Q \operatorname{coh}(X)$ where all $\mathcal{I}_{i}$ are injective. If we restrict this sequence to an arbitrary affine open subset $U$ of $X$, the affine case treated above shows that $\left.\mathcal{C}\right|_{U}$ is injective in $\mathrm{Qcoh}(U)$. Hence $\mathcal{C}$ is injective in $\mathrm{Q} \operatorname{coh}(X)$. This shows that the global dimension of $\mathrm{Qcoh}(X)$ is $\leq d$. Similarly as above, it must in fact be equal to $d$.

Remark 5.13. Let $\mathcal{A}$ be a locally noetherian Grothendieck category and let $\mathcal{U} \subset \mathcal{A}$ be the full subcategory of noetherian objects. Then the global dimensions of the abelian categories $\mathcal{U}$ and $\mathcal{A}$ coincide. Indeed, the argument of the first paragraph of the proof of Theorem 5.10 shows that the global dimension of $\mathcal{U}$ cannot exceed the global dimension of $\mathcal{A}$. The converse inequality is provable using the next two lemmas.

Lemma 5.14. An object $J \in \mathcal{A}$ is injective if and only if, for every object $U \in \mathcal{U}$ and every subobject $V \hookrightarrow U$, any morphism $V \rightarrow J$ can be extended to a morphism $U \rightarrow J$.

Proof. The non-trivial implication is a standard Zorn lemma argument very similar to the proof of the Baer criterion for injectivity of modules. Given an object $A \in \mathcal{A}$, a subobject $B \hookrightarrow A$, and a morphism $B \rightarrow J$, one considers the poset of all morphisms $C \rightarrow J$ defined on intermediate subobjects $B \hookrightarrow C \hookrightarrow A$ and making the triangular diagram $B \hookrightarrow C \rightarrow J$ commutative. This poset clearly has unions of chains, and therefore it has a maximal element $C \rightarrow J$. We claim that $C=A$. If not, there is a noetherian subobject $U \hookrightarrow A$ that is not contained in $C$. The restriction of the morphism $C \rightarrow J$ to the subobject $V=U \cap C \hookrightarrow C$ has by assumption an extension along the inclusion $V \hookrightarrow U$ to a morphism $U \rightarrow J$. The two morphisms $C \rightarrow J$ and $U \rightarrow J$ agree on the intersection $U \cap C$ and can therefore be uniquely extended to a morphism $C+U \rightarrow J$. Since $C \subsetneq C+U$ we obtain a contradiction.

Lemma 5.15. For any object $U \in \mathcal{U}$ and every integer $n \geq 0$, the functor $\operatorname{Ext}_{\mathcal{A}}^{n}(U,-)$ takes filtered colimits in $\mathcal{A}$ to filtered colimits of abelian groups.

Proof. Given an object $A \in \mathcal{A}$, the abelian group $\operatorname{Ext}_{\mathcal{A}}^{n}(U, A)=\operatorname{Hom}_{\mathrm{D}(\mathcal{A})}\left(U, \Sigma^{n} A\right)$ can be computed as the set of all equivalence classes of roofs $U \leftarrow P \rightarrow \Sigma^{n} A$, where $P \rightarrow U$ is a quasi-isomorphism of complexes in $\mathcal{A}$. Clearly, it suffices to consider bounded above complexes $P$. Following the proof of Proposition 2.1, we can assume that $P$ is a complex in $\mathcal{U}$. Recall from [Gab62, II.4, Corollaire 1] that, given any object $V \in \mathcal{U}$, the functor $\operatorname{Hom}_{\mathcal{A}}(V,-)$ takes filtered colimits in $\mathcal{A}$ to filtered colimits of abelian groups. If now $A=\operatorname{colim}_{i \in I} A_{i}$ is a filtered colimit in $\mathcal{A}$, it is straightforward to see that the natural map $\operatorname{colim}_{i \in I} \operatorname{Hom}_{\mathrm{K}(\mathcal{A})}\left(P, \Sigma^{n} A_{i}\right) \rightarrow \operatorname{Hom}_{\mathrm{K}(\mathcal{A})}\left(P, \Sigma^{n} A\right)$ is bijective, for all complexes $P$ in $\mathcal{U}$; we deduce that the canonical map $\operatorname{colim}_{i \in I} \operatorname{Hom}_{\mathrm{D}(\mathcal{A})}\left(U, \Sigma^{n} A_{i}\right) \rightarrow \operatorname{Hom}_{\mathrm{D}(\mathcal{A})}(U, A)$ is bijective as well. 
Alternatively, one can devise an argument based on the fact that the class of injective objects in any locally noetherian Grothendieck category is closed under filtered colimits (see [Gab62, II.4, Corollaire 1]). Given a diagram $\left(A_{i}\right)_{i \in I}$ in $\mathcal{A}$ indexed by a directed poset $I$, one then needs to construct an $I$-indexed diagram of injective resolutions $A_{i} \rightarrow J_{i}$ of the objects $A_{i}$. To this end, one can observe that functorial injective resolutions exist in any Grothendieck category (see e.g. [SP20, 079H]). One can also consider the Grothendieck category $\mathcal{A}^{I}$ of $I$-indexed diagrams in $\mathcal{A}$ and notice that any injective object in $\mathcal{A}^{I}$ is a diagram of injective objects in $\mathcal{A}$, because the functor $\mathcal{A}^{I} \rightarrow \mathcal{A}$ taking a diagram $\left(K_{i}\right)_{i \in I}$ to the object $K_{j}$ for a fixed index $j \in I$ is right adjoint to an exact functor $\mathcal{A} \rightarrow \mathcal{A}^{I}$ and consequently takes injectives to injectives. So any injective resolution of $\left(A_{i}\right)_{i \in I}$ in $\mathcal{A}^{I}$ provides the desired diagram of injective resolutions.

Then $\operatorname{colim}_{i \in I} J_{i}$ is an injective resolution of the object $\operatorname{colim}_{i \in I} A_{i}$ in $\mathcal{A}$. Computing $\operatorname{Ext}_{\mathcal{A}}^{n}\left(U, A_{i}\right)$ in terms of the injective resolution $J_{i}$ of the object $A_{i}$ and $\operatorname{Ext}_{\mathcal{A}}^{n}\left(U, \operatorname{colim}_{i \in I} A_{i}\right)$ in terms of the injective resolution $\operatorname{colim}_{i \in I} J_{i}$ of the object colim ${ }_{i \in I} A_{i}$, and using the fact that the functor $\operatorname{Hom}_{\mathcal{A}}(U,-)$ takes filtered colimits in $\mathcal{A}$ to filtered colimits of abelian groups (see [Gab62, II.4, Corollaire 1]), one deduces the same property for the functor $\operatorname{Ext}_{\mathcal{A}}^{n}(U,-)$.

Now we can finish the proof of the converse inequality. Let $d$ be the global dimension of $\mathcal{U}$. If $d=\infty$, then there is nothing to prove. The case $d=-1$ is also trivial. So we assume that $d \geq 0$ is a natural number. Then we have $\operatorname{Ext}_{\mathcal{A}}^{d+1}(U, W)=0$ for all $U, W \in \mathcal{U}$. Let $A \in \mathcal{A}$ be arbitrary. Since $A$ is the filtered colimit of its noetherian subobjects, Lemma 5.15 shows that $\operatorname{Ext}_{\mathcal{A}}^{d+1}(U, A)=0$ for all $U \in \mathcal{U}$. Let $J$ be an injective resolution of $A$ in $\mathcal{A}$, and let $Z^{d}$ be the kernel of the differential $J^{d} \rightarrow J^{d+1}$. Then $\operatorname{Ext}_{\mathcal{A}}^{1}\left(U, Z^{d}\right)=\operatorname{Ext}_{\mathcal{A}}^{d+1}(U, A)=0$ for all $U \in \mathcal{U}$. By Lemma 5.14, we conclude that the object $Z^{d} \in \mathcal{A}$ is injective. This implies that the global dimension of $\mathcal{A}$ is $\leq d$.

\section{Appendix A. The hereditary CASE}

The aim of this appendix is to give a full proof of Corollary A.2. This is an easy consequence of Theorem A.1, a structural result on the unbounded derived category of a hereditary abelian category.

If $A$ and $B$ are objects of an abelian category $\mathcal{A}$, we may view them as complexes concentrated in degree zero and then have a canonical identification $\operatorname{Hom}_{\mathcal{A}}(A, B)=$ $\operatorname{Hom}_{\mathrm{D}(\mathcal{A})}(A, B)$. By definition, we have $\operatorname{Ext}_{\mathcal{A}}^{n}(A, B)=\operatorname{Hom}_{\mathrm{D}(\mathcal{A})}\left(A, \Sigma^{n} B\right)$ for $n \in \mathbb{Z}$. We remind the reader that an abelian category is called hereditary if and only if $\operatorname{Ext}_{\mathcal{A}}^{2}(A, B)=0$ for all objects $A, B$ of $\mathcal{A}$.

Theorem A.1 (cf. [Kra07, Section 1.6]). Let $\mathcal{A}$ be a hereditary abelian category. Then any object $M$ in $\mathrm{D}(\mathcal{A})$ is isomorphic to a complex with vanishing differentials whose $n$-th component is then necessarily isomorphic to $\mathrm{H}^{n}(M)$.

Let $X=\left(\left(X^{n}\right), 0\right)$ and $Y=\left(\left(Y^{n}\right), 0\right)$ be complexes in $\mathcal{A}$ with vanishing differentials. Let $\iota_{n}: \Sigma^{-n} X^{n} \rightarrow X$ and $\pi_{n}: Y \rightarrow \Sigma^{-n} Y^{n}$ be the obvious inclusion and projection morphisms of complexes. Then the map

$$
\begin{aligned}
\operatorname{Hom}_{\mathrm{D}(\mathcal{A})}(X, Y) & \stackrel{\sim}{\rightarrow} \prod_{n \in \mathbb{Z}} \operatorname{Hom}_{\mathcal{A}}\left(X^{n}, Y^{n}\right) \times \prod_{n \in \mathbb{Z}} \operatorname{Ext}_{\mathcal{A}}^{1}\left(X^{n}, Y^{n-1}\right), \\
f & \mapsto\left(\left(\Sigma^{n}\left(\pi_{n} \circ f \circ \iota_{n}\right)\right)_{n \in \mathbb{Z}},\left(\Sigma^{n}\left(\pi_{n-1} \circ f \circ \iota_{n}\right)\right)_{n \in \mathbb{Z}}\right),
\end{aligned}
$$

is bijective (note that $\left.\Sigma^{n}\left(\pi_{n} \circ f \circ \iota_{n}\right)=\mathrm{H}^{n}(f)\right)$. 
In particular, any complex $Z=\left(\left(Z^{n}\right), 0\right)$ in $\mathcal{A}$ with vanishing differentials is both coproduct and product in $\mathrm{D}(\mathcal{A})$ of the objects $\Sigma^{-n} Z^{n}$, for $n \in \mathbb{Z}$, with obvious inclusion and projection morphisms.

Altogether we have $\bigoplus_{n \in \mathbb{Z}} \Sigma^{-n} \mathrm{H}^{n}(M) \cong M \cong \prod_{n \in \mathbb{Z}} \Sigma^{-n} \mathrm{H}^{n}(M)$ for any object $M$ of $\mathrm{D}(\mathcal{A})$.

Proof. The first part of the proof is copied from [Kra07, Section 1.6]. The second part, bijectivity of (A.1), is treated rather quickly in loc. cit.; we therefore provide full details.

Let $M$ be a complex in $\mathcal{A}$. For $n \in \mathbb{Z}$, the epimorphism $d^{\prime}=d^{\prime n-1}: M^{n-1} \rightarrow$ $\mathrm{B}^{n}(M)$ induced by $d=d^{n-1}: M^{n-1} \rightarrow M^{n}$ yields a surjection $\operatorname{Ext}_{\mathcal{A}}^{1}\left(\mathrm{H}^{n}(M), M^{n-1}\right) \rightarrow$ $\operatorname{Ext}_{\mathcal{A}}^{1}\left(\mathrm{H}^{n}(M), \mathrm{B}^{n}(M)\right)$ because $\operatorname{Ext}_{\mathcal{A}}^{2}$ vanishes. Hence the extension $0 \rightarrow \mathrm{B}^{n}(M) \stackrel{i=i^{n}}{\longrightarrow}$ $\mathrm{Z}^{n}(M) \stackrel{p=p^{n}}{\longrightarrow} \mathrm{H}^{n}(M) \rightarrow 0$ comes from an extension $0 \rightarrow M^{n-1} \stackrel{j=j^{n}}{\longrightarrow} E^{n} \stackrel{q=q^{n}}{\longrightarrow} \mathrm{H}^{n}(M) \rightarrow$ 0 , i. e. there is a commutative diagram

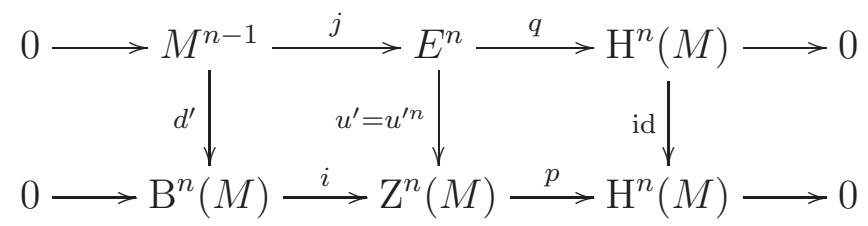

in $\mathcal{A}$ whose left square is cocartesian. It is easy to check that the diagram

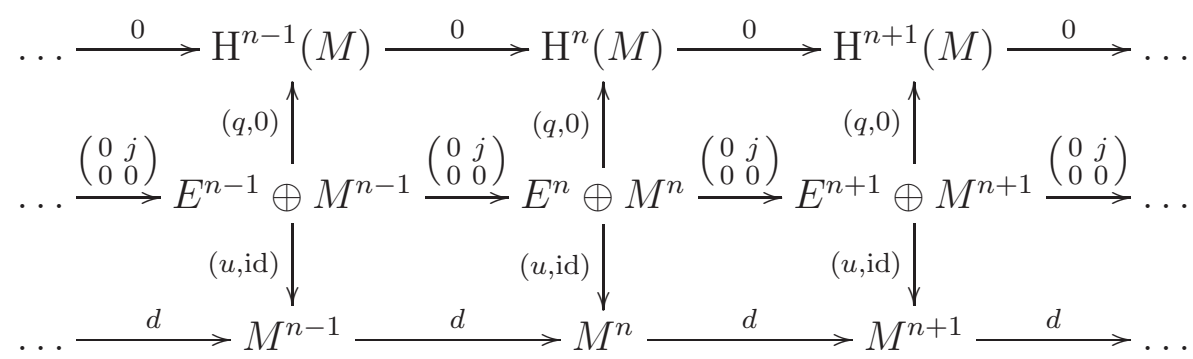

in $\mathcal{A}$ is commutative where $u=u^{n}$ is the composition $E^{n} \stackrel{u^{\prime}}{\rightarrow} \mathrm{Z}^{n}(M) \hookrightarrow M^{n}$. The rows are complexes and the vertical arrows define quasi-isomorphisms between these complexes. This proves the first claim.

We now prove surjectivity of (A.1). Assume that morphisms $g_{n}: X^{n} \rightarrow Y^{n}$ in $\mathcal{A}$ and $e_{n}: X^{n} \rightarrow \Sigma Y^{n-1}$ in $\mathrm{D}(\mathcal{A})$ are given, for all $n \in \mathbb{Z}$. Represent $e_{n}$ by a short exact sequence $0 \rightarrow Y^{n-1} \stackrel{k=k^{n}}{\longrightarrow} F^{n} \stackrel{r=r^{n}}{\longrightarrow} X^{n} \rightarrow 0$ and consider the commutative diagram

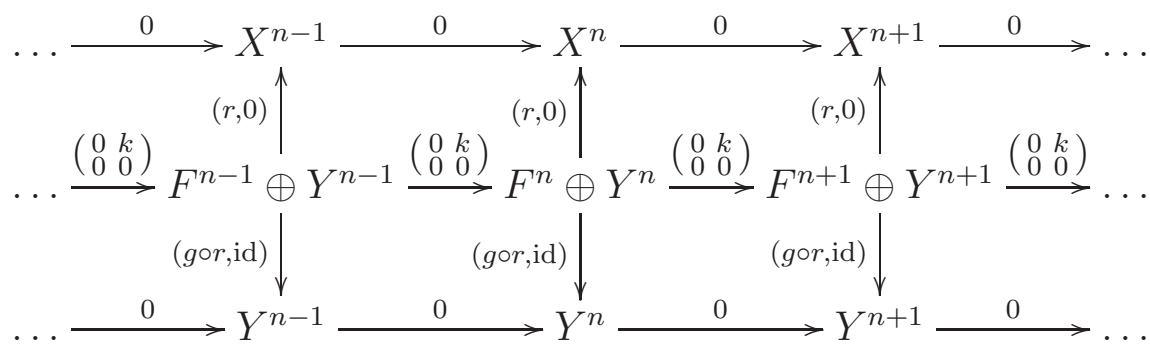

whose rows are complexes. The vertical arrows define morphisms of complexes, and the upper morphism $(r, 0)$ is a quasi-isomorphism. We interprete this diagram as a roof which gives rise to a morphism $f: X \rightarrow Y$ in $\mathrm{D}(\mathcal{A})$. This morphism $f$ is mapped to the element $\left(\left(g_{n}\right),\left(e_{n}\right)\right)$; to see this, use the subcomplex $\ldots \rightarrow 0 \rightarrow Y^{n-1} \stackrel{k}{\rightarrow} F^{n} \rightarrow 0 \rightarrow \ldots$ of the middle row together with its quasi-isomorphism to the subcomplex $\Sigma^{-n} X^{n}$ of the upper row. 
We now prepare for the proof of injectivity of (A.1). Let $f: X \rightarrow Y$ be any morphism in $\mathrm{D}(\mathcal{A})$. Represent $f$ by a roof $X \stackrel{a}{\leftarrow} M \stackrel{b}{\rightarrow} Y$ where $M$ is a suitable complex in $\mathcal{A}$, both $a$ and $b$ are morphisms of complexes and $a$ is a quasi-isomorphism. We deal with $M$ as explained in the first part of this proof. By precomposing with the quasi-isomorphism $(u$,id) between middle and lower row in (A.2) we obtain the representation of $f$ by the roof

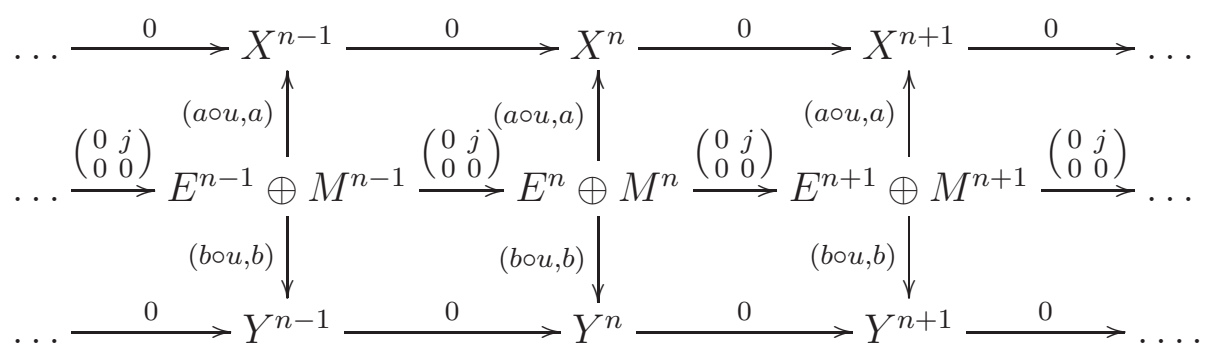

Commutativity of the upper squares shows $a \circ u \circ j=a^{n} \circ u^{n} \circ j^{n}=0$ and hence $a \circ u=\bar{a} \circ q$ for a unique morphism $\bar{a}=\bar{a}^{n}: \mathrm{H}^{n}(M) \rightarrow X^{n}$. Since $a$ is a quasi-isomorphism, $\bar{a}=\bar{a}^{n}$ is an isomorphism. Commutativity of the lower squares shows that $b \circ u \circ j=b^{n} \circ u^{n} \circ j^{n}=0$ and hence $b \circ u=\bar{b} \circ q$ for a unique morphism $\bar{b}=\bar{b}^{n}: \mathrm{H}^{n}(M) \rightarrow Y^{n}$.

Using the subcomplex $\ldots \rightarrow 0 \rightarrow M^{n-1} \stackrel{j}{\rightarrow} E^{n} \rightarrow 0 \rightarrow \ldots$ of the middle row of (A.3) together with its quasi-isomorphism to $\Sigma^{-n} X^{n}$ we first see that

$$
\Sigma^{n}\left(\pi_{n} \circ f \circ \iota_{n}\right)=\bar{b} \circ \bar{a}^{-1}: X^{n} \stackrel{\bar{a}^{-1}}{\sim} \mathrm{H}^{n}(M) \stackrel{\bar{b}}{\rightarrow} Y^{n} .
$$

Second we see that $\pi_{n-1} \circ f \circ \iota_{n}$ is given by the roof

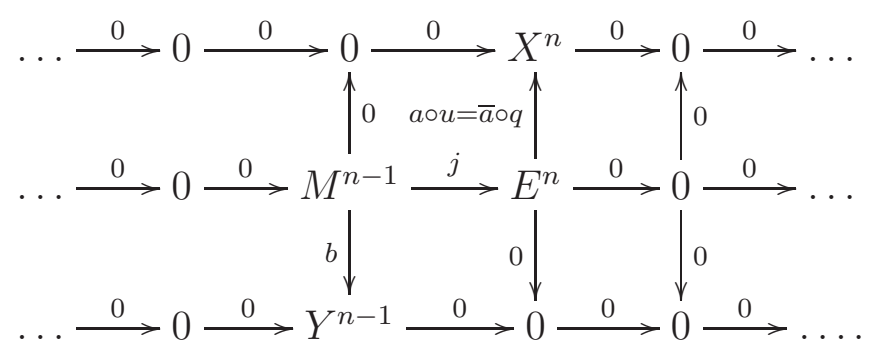

Consider the morphism of short exact sequences

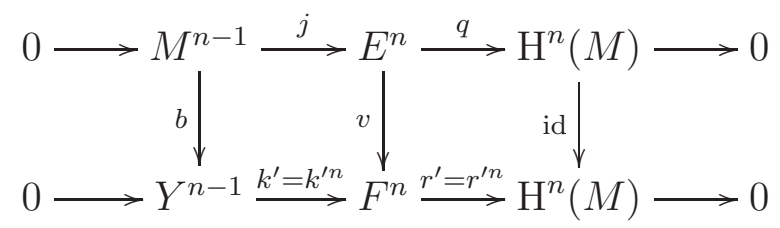

where the left square is cocartesian. The lower row is a Yoneda extension representing $\Sigma^{n}\left(\pi_{n-1} \circ f \circ \iota_{n}\right)$ if we identify $\mathrm{H}^{n}(M) \underset{\sim}{\stackrel{\bar{a}}{\longrightarrow}} X^{n}$.

The morphism $(b \circ u, b)$ between the lower two rows in (A.3) factors as the composition of the following two morphisms of complexes (where the first morphism is a 
quasi-isomorphism).

$$
\begin{aligned}
& \ldots \stackrel{\left(\begin{array}{ll}
0 & j \\
0 & 0
\end{array}\right)}{\longrightarrow} E^{n-1} \oplus M^{n-1} \stackrel{\left(\begin{array}{ll}
0 & j \\
0 & 0
\end{array}\right)}{\longrightarrow} E^{n} \oplus M^{n} \stackrel{\left(\begin{array}{ll}
0 & j \\
0 & 0
\end{array}\right)}{\longrightarrow} E^{n+1} \oplus M^{n+1} \stackrel{\left(\begin{array}{ll}
0 & j \\
0 & 0
\end{array}\right)}{\longrightarrow} \ldots
\end{aligned}
$$

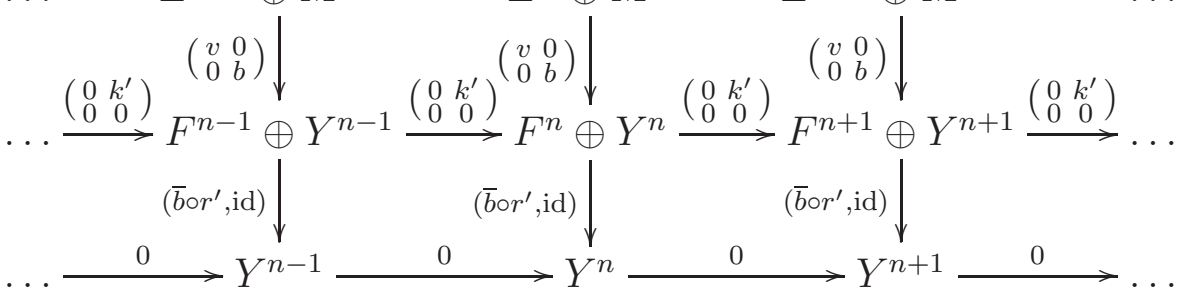

In order to show injectivity of (A.1) assume now that $f$ is mapped to zero. First $0=\Sigma^{n}\left(\pi_{n} \circ f \circ \iota_{n}\right)=\bar{b} \circ \bar{a}^{-1}$ yields $\bar{b}=0$. Hence $\bar{b} \circ r=0$, i. e. the lower vertical morphism $\left(\bar{b} \circ r^{\prime}\right.$, id) in $(\mathrm{A} .5)$ equals $(0, \mathrm{id})$.

Second $\Sigma^{n}\left(\pi_{n-1} \circ f \circ \iota_{n}\right)=0$ means that the extension in the lower row of (A.4) is trivial. Hence we can assume that $F^{n}=Y^{n-1} \oplus \mathrm{H}^{n}(M)$ and that $k^{\prime}=\left(\begin{array}{c}\text { id } \\ 0\end{array}\right)$ and $r^{\prime}=(0$, id $)$. Using this, the morphism $\left(\bar{b} \circ r^{\prime}\right.$, id $)=(0$, id $)$ in $($ A.5) has the form

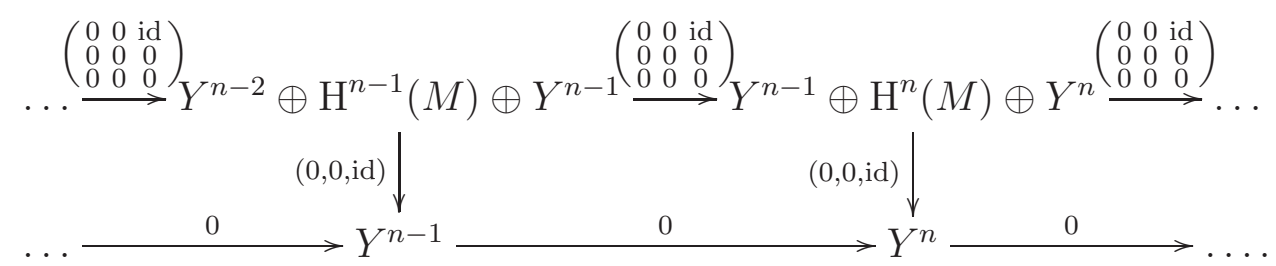

But this morphism $(0,0$, id) is zero in the homotopy category $\mathrm{K}(\mathcal{A})$ (take the homotopy $(\mathrm{id}, 0,0))$. This shows that the morphism $\left(\bar{b} \circ r^{\prime}, \mathrm{id}\right)$ vanishes in $\mathrm{K}(\mathcal{A})$. Hence $(b \circ u, b)$ vanishes in $\mathrm{K}(\mathcal{A})$ and $f$ vanishes in $\mathrm{D}(\mathcal{A})$. This shows that the map (A.1) is bijective. From this the remaining claims follow immediately.

Corollary A.2. Let $\mathcal{A}$ be a hereditary abelian category and let $\mathcal{U} \subset \mathcal{A}$ be a non-empty, full subcategory closed under kernels, cokernels, and extensions. Then the obvious functor is an equivalence $\mathrm{D}(\mathcal{U}) \stackrel{\sim}{\rightarrow} \mathrm{D}_{\mathcal{U}}(\mathcal{A})$ of triangulated categories.

Proof. This follows from Theorem A.1. First, the essential image of the functor $\mathrm{D}(\mathcal{U}) \rightarrow$ $\mathrm{D}(\mathcal{A})$ is $\mathrm{D}_{\mathcal{U}}(\mathcal{A})$. Second, fully faithfulness can be tested on objects $X, Y$ of $\mathrm{D}(\mathcal{U})$ with vanishing differentials, and for those the map $\operatorname{Hom}_{\mathrm{D}(\mathcal{U})}(X, Y) \rightarrow \operatorname{Hom}_{\mathrm{D}(\mathcal{A})}(X, Y)$ is identified with the obvious map

$$
\prod_{n \in \mathbb{Z}} \operatorname{Hom}_{\mathcal{U}}\left(X^{n}, Y^{n}\right) \times \prod_{n \in \mathbb{Z}} \operatorname{Ext}_{\mathcal{U}}^{1}\left(X^{n}, Y^{n-1}\right) \rightarrow \prod_{n \in \mathbb{Z}} \operatorname{Hom}_{\mathcal{A}}\left(X^{n}, Y^{n}\right) \times \prod_{n \in \mathbb{Z}} \operatorname{Ext}_{\mathcal{A}}^{1}\left(X^{n}, Y^{n-1}\right)
$$

which is an isomorphism by our assumption that $\mathcal{U}$ is a full subcategory of $\mathcal{A}$ that is closed under extensions.

\section{REFERENCES}

[AF92] Frank W. Anderson and Kent R. Fuller. Rings and categories of modules, volume 13 of Graduate Texts in Mathematics. Springer-Verlag, New York, second edition, 1992.

[ARS97] Maurice Auslander, Idun Reiten, and Sverre O. Smalø. Representation theory of Artin algebras, volume 36 of Cambridge Studies in Advanced Mathematics. Cambridge University Press, Cambridge, 1997. Corrected reprint of the 1995 original.

[Aus55] Maurice Auslander. On the dimension of modules and algebras. III. Global dimension. Nagoya Math. J., 9:67-77, 1955.

[Gab62] Pierre Gabriel. Des catégories abéliennes. Bull. Soc. Math. France, 90:323-448, 1962. 
[Har66] Robin Hartshorne. Residues and duality. Lecture notes of a seminar on the work of A. Grothendieck, given at Harvard 1963/64. With an appendix by P. Deligne. Lecture Notes in Mathematics, No. 20. Springer-Verlag, Berlin, 1966.

[Huy06] Daniel Huybrechts. Fourier-Mukai transforms in algebraic geometry. Oxford Mathematical Monographs. The Clarendon Press Oxford University Press, Oxford, 2006.

[Kel98] Bernhard Keller. On the construction of triangle equivalences. In Derived equivalences for group rings, volume 1685 of Lecture Notes in Math., pages 155-176. Springer, Berlin, 1998.

[Kra07] Henning Krause. Derived categories, resolutions, and Brown representability. In Interactions between homotopy theory and algebra, volume 436 of Contemp. Math., pages 101-139. Amer. Math. Soc., Providence, RI, 2007.

[KS94] Masaki Kashiwara and Pierre Schapira. Sheaves on manifolds, volume 292 of Grundlehren der Mathematischen Wissenschaften. Springer-Verlag, Berlin, 1994.

[KS06] Masaki Kashiwara and Pierre Schapira. Categories and sheaves, volume 332 of Grundlehren der Mathematischen Wissenschaften. Springer-Verlag, Berlin, 2006.

[Lam99] T. Y. Lam. Lectures on modules and rings, volume 189 of Graduate Texts in Mathematics. Springer-Verlag, New York, 1999.

[LC07] Jue Le and Xiao-Wu Chen. Karoubianness of a triangulated category. J. Algebra, 310(1):452457, 2007.

[LS16] Valery A. Lunts and Olaf M. Schnürer. Matrix factorizations and semi-orthogonal decompositions for blowing-ups. J. Noncommut. Geom., 10(3):907-979, 2016.

[Mat89] Hideyuki Matsumura. Commutative ring theory, volume 8 of Cambridge Studies in Advanced Mathematics. Cambridge University Press, Cambridge, second edition, 1989.

[NY03] W. K. Nicholson and M. F. Yousif. Quasi-Frobenius rings, volume 158 of Cambridge Tracts in Mathematics. Cambridge University Press, Cambridge, 2003.

[Pos17] Leonid Positselski. Contraherent cosheaves, 2012-17. arXiv:1209.2995 [math.CT].

[Pos10] Leonid Positselski. Homological algebra of semimodules and semicontramodules: Semi-infinite homological algebra of associative algebraic structures, volume 70 of Monografie Matematyczne (New Series), Instytut Matematyczny Polskiej Akademii Nauk. Birkhäuser/Springer Basel AG, Basel, 2010. Appendix C in collaboration with Dmitriy Rumynin; Appendix D in collaboration with Sergey Arkhipov. arXiv:0708.3398 [math.CT].

[Pos11] Leonid Positselski. Two kinds of derived categories, Koszul duality, and comodule-contramodule correspondence. Mem. Amer. Math. Soc., 212(996):vi+133, 2011. arXiv:0905.2621 [math.CT].

[Rot09] Joseph J. Rotman. An introduction to homological algebra. Universitext. Springer, New York, second edition, 2009.

[Sch11] Olaf M. Schnürer. Homotopy categories and idempotent completeness, weight structures and weight complex functors, 2011. arXiv:1107.1227v1 [math.CT].

[SGA6] P. Berthelot, A. Grothendieck, and L. Illusie. Théorie des intersections et théorème de Riemann-Roch. Lecture Notes in Mathematics, Vol. 225. Springer-Verlag, Berlin, 1971. Séminaire de Géométrie Algébrique du Bois-Marie 1966-1967 (SGA 6).

[SP20] The Stacks Project Authors. The Stacks Project. http://stacks.math.columbia.edu, 2020.

Institute of Mathematics of the Czech Academy of Sciences, Žitná 25, 11567 Prague 1 , Czech Republic; And

Laboratory of Algebra and Number Theory, Institute for Information Transmission Problems, Moscow 127051, Russia

Email address: positselski@math.cas.cz

Institut für Mathematik, Universität Paderborn, Warburger Strasse 100, 33098 PaDERBorn, GERMANY

Email address: olaf.schnuerer@math.uni-paderborn.de 